\title{
Hyperchaos, adaptive control and synchronization of a novel 5-D hyperchaotic system with three positive Lyapunov exponents and its SPICE implementation
}

\author{
SUNDARAPANDIAN VAIDYANATHAN, CHRISTOS VOLOS and VIET-THANH PHAM
}

\begin{abstract}
In this research work, a twelve-term novel 5-D hyperchaotic Lorenz system with three quadratic nonlinearities has been derived by adding a feedback control to a ten-term 4-D hyperchaotic Lorenz system (Jia, 2007) with three quadratic nonlinearities. The 4-D hyperchaotic Lorenz system (Jia, 2007) has the Lyapunov exponents $L_{1}=0.3684, L_{2}=0.2174, L_{3}=0$ and $L_{4}=-12.9513$, and the Kaplan-Yorke dimension of this 4-D system is found as $D_{K Y}=3.0452$. The 5-D novel hyperchaotic Lorenz system proposed in this work has the Lyapunov exponents $L_{1}=0.4195, L_{2}=0.2430, L_{3}=0.0145, L_{4}=0$ and $L_{5}=-13.0405$, and the Kaplan-Yorke dimension of this 5-D system is found as $D_{K Y}=4.0159$. Thus, the novel 5-D hyperchaotic Lorenz system has a maximal Lyapunov exponent (MLE), which is greater than the maximal Lyapunov exponent (MLE) of the 4-D hyperchaotic Lorenz system. The 5-D novel hyperchaotic Lorenz system has a unique equilibrium point at the origin, which is a saddle-point and hence unstable. Next, an adaptive controller is designed to stabilize the novel 5-D hyperchaotic Lorenz system with unknown system parameters. Moreover, an adaptive controller is designed to achieve global hyperchaos synchronization of the identical novel 5-D hyperchaotic Lorenz systems with unknown system parameters. Finally, an electronic circuit realization of the novel 5-D hyperchaotic Lorenz system using SPICE is described in detail to confirm the feasibility of the theoretical model.
\end{abstract}

Key words: chaos, hyperchaos, control, synchronization, circuit realization

\section{Introduction}

Nonlinear dynamics occurs widely in engineering, physics, biology and many other scientific disciplines [1]. Poincaré was the first to observe the possibility of chaos, in which a deterministic system exhibits aperiodic behavior that depends on the initial conditions, thereby rendering long-term prediction impossible, since then it has received much attention $[2,3]$. Interest in nonlinear dynamics and in particular chaotic dynamics

S. Vaidyanathan, the corresponding author is with the Research and Development Centre, Vel Tech University, Avadi, Chennai- 600062, Tamilnadu, India. E-mail: sundarvtu@gmail.com. Ch. Volos is with Physics Department, Aristotle University of Thessaloniki, GR-54124, Greece. V-T. Pham is with School of Electronics and Telecommunications, Hanoi University of Science and Technology, 01 Dai Co Viet, Hanoi, Vietnam.

Received 29.09.2014. 
has grown rapidly since 1963, when Lorenz published his numerical work on a simplified model of convection and discussed its implications for weather prediction [4].

Chaos theory describes the qualitative study of unstable aperiodic behavior in deterministic nonlinear dynamical systems. For the motion of a dynamical system to be chaotic, the system variables should contain nonlinear terms and it must satisfy three properties: boundedness, infinite recurrence and sensitive dependence on initial conditions [5].

The Lyapunov exponent of a dynamical system is a quantity that characterizes the rate of separation of infinitesimally close trajectories. The sensitive dependence on initial conditions of a dynamical system is characterized by the presence of a positive Lyapunov exponent. A positive Lyapunov exponent reflects a direction of stretching and folding and along with phase-space compactness indicates the presence of chaos in a dynamical system. An $n$-dimensional dynamical system has a spectrum of $n$ Lyapunov exponents and the maximal Lyapunov exponent (MLE) of a chaotic system is defined as the largest positive Lyapunov exponent of the system.

Chaos has developed over time. For example, Ruelle and Takens [6] proposed a theory for the onset of turbulence in fluids, based on abstract considerations about strange attractors. Later, May [7] found examples of chaos in iterated mappings arising in population biology. Feigenbaum [8] discovered that there are certain universal laws governing the transition from regular to chaotic behaviours. That is, completely different systems can go chaotic in the same way, thus, linking chaos and phase transitions.

The first famous chaotic system was accidentally discovered by Lorenz, when he was designing a 3-D model for atmospheric convection in 1963 [9]. Subsequently, Rössler discovered a 3-D chaotic system in 1976 [10], which is algebraically simpler than the Lorenz system. Indeed, Lorenz's system is a seven-term chaotic system with two quadratic nonlinearities, while Rössler's system is a seven-term chaotic system with just one quadratic nonlinearity.

Some well-known paradigms of 3-D chaotic systems are Arneodo system [11], Sprott systems [12], Chen system [13], Lü-Chen system [14], Liu system [15], Cai system [16], T-system [17], etc. Many new chaotic systems have been also discovered like Li system [18], Sundarapandian systems [19, 20], Vaidyanathan systems [21, 22, 23, 24, 25, 26, 27, 28], Pehlivan system [29], Jafari system [30], Pham system [31], etc.

Chaos theory has applications in several fields of science and engineering such as oscillators [32, 33, 34], lasers [35, 36, 37], chemical reactions [38, 39, 40], biology [41, 42], ecology [43, 44, 45], neural networks [46, 47, 48, 49], robotics [50, 51, 52], fuzzy logic [53, 54], electrical circuits [55, 56, 57], etc.

A hyperchaotic system is generally defined as a chaotic system with at least two positive Lyapunov exponents [58]. Thus, the dynamics of a hyperchaotic system are expended in several different directions simultaneously. Thus, the hyperchaotic systems have more complex dynamical behaviour and hence they have miscellaneous applications in engineering such as secure communications [59, 60, 61], cryptosystems $[62,63,64]$, encryption $[65,66,67]$, electrical circuits $[68,69,70,71]$, etc. 
The minimum dimension for an autonomous, continuous-time, hyperchaotic system is four. Since the discovery of a first 4-D hyperchaotic system by Rössler in 1979 [72], many 4-D hyperchaotic systems have been found in the literature such as hyperchaotic Lorenz system [73], hyperchaotic Lü system [74], hyperchaotic Chen system [75], hyperchaotic Wang system [76], hyperchaotic Newton-Leipnik system [77], hyperchaotic Vaidyanathan system [78], etc.

Recently, there is some considerable interest in finding novel 5-D hyperchaotic systems with three positive Lyapunov exponents and such 5-D hyperchaotic systems have been found in the literature such as hyperchaotic Hu system [79], [80], etc.

In this research work, a twelve-term novel 5-D hyperchaotic Lorenz system with three quadratic nonlinearities has been derived by adding a feedback control to a 4-D hyperchaotic Lorenz system with three quadratic nonlinearities [81]. The 4-D hyperchaotic Lorenz system [81] has the Lyapunov exponents $L_{1}=0.3684, L_{2}=0.2174, L_{3}=0$ and $L_{4}=-12.9513$, and the Kaplan-Yorke dimension of this 4-D system is found as $D_{K Y}=3.0452$. The 5-D novel hyperchaotic Lorenz system proposed in this work has the Lyapunov exponents $L_{1}=0.4195, L_{2}=0.2430, L_{3}=0.0145, L_{4}=0$ and $L_{5}=-13.0405$. The Kaplan-Yorke dimension of the 5-D novel hyperchaotic Lorenz system is found as $D_{K Y}=4.0159$. Thus, the novel 5-D novel hyperchaotic Lorenz system has a maximal Lyapunov exponent (MLE), which is greater than the maximal Lyapunov exponent (MLE) of the 4-D hyperchaotic Lorenz system.

In this work, adaptive control and synchronization schemes have been also developed for the novel 5-D hyperchaotic Lorenz system with three positive Lyapunov exponents.

The study of control of a chaotic system investigates methods for designing feedback control laws that globally or locally asymptotically stabilize or regulate the outputs of a chaotic system.

Many methods have been developed for the control and tracking of chaotic systems such as active control [82, 83, 84, 85], adaptive control [86, 87, 88, 89, 90, 91, 92], backstepping control [93, 94, 95], sliding mode control [96, 97], etc.

Chaos synchronization problem deals with the synchronization of a couple of systems called the master or drive system and the slave or response system. To solve this problem, control laws are designed so that the output of the slave system tracks the output of the master system asymptotically with time.

The study of chaos in the last decades had a tremendous impact on the foundations of science and engineering and one of the most recent exciting developments in this regard is the discovery of chaos synchronization, whose possibility was first reported by Fujisaka and Yamada [98] and later by Pecora and Carroll [99].

Because of the butterfly effect, the synchronization of chaotic systems is a challenging problem in the chaos literature even when the initial conditions of the master and slave systems are nearly identical because of the exponential divergence of the outputs of the two systems in the absence of any control.

Different types of synchronization such as complete synchronization [99], antisynchronization [100, 101, 102], hybrid synchronization [103, 104], lag synchronization [105], phase synchronization [105, 106], anti-phase synchronization [107], gener- 
alized synchronization [108], projective synchronization [109], generalized projective synchronization $[110,111,112]$, etc. have been studied in the chaos literature.

Since the discovery of chaos synchronization, different approaches have been proposed to achieve it, such as PC method [99], active control method [113, 114, 115, 116], adaptive control method $[117,118,119,120]$, backstepping control method [121, 122, $123,124,125,126]$, sliding mode control method [127, 128, 129, 130, 131], etc.

All the main adaptive results in this paper are proved using Lyapunov stability theory. MATLAB simulations are depicted to illustrate the phase portraits of the novel 5-D hyperchaotic Lorenz system with three positive Lyapunov exponents, adaptive stabilization and synchronization results for the novel 5-D hyperchaotic Lorenz system.

Finally, an electronic circuit realization of the novel 5-D hyperchaotic Lorenz system using SPICE is presented to confirm the feasibility of the theoretical model.

\section{A 5-D novel hyperchaotic Lorenz system}

In [81], Jia (2007) obtained a 4-D hyperchaotic Lorenz system by adding a feedback control to the famous Lorenz system [9], and this 4-D system is given by the dynamics

$$
\left\{\begin{array}{l}
\dot{x}_{1}=a\left(x_{2}-x_{1}\right)+x_{4} \\
\dot{x}_{2}=c x_{1}-x_{1} x_{3}-x_{2} \\
\dot{x}_{3}=x_{1} x_{2}-b x_{3} \\
\dot{x}_{4}=-x_{1} x_{3}+p x_{4}
\end{array}\right.
$$

where $x_{1}, x_{2}, x_{3}, x_{4}$ are the system parameters and $a, b, c, p$ are positive, constant, parameters.

In [81], it was shown that the 4-D system (1) is hyperchaotic when the parameter values are taken as

$$
a=10, b=8 / 3, c=28, p=1.3
$$

Also, for these parameter values, the Lyapunov exponents of the 4-D hyperchaotic Lorenz system (1) are calculated as

$$
L_{1}=0.3684, L_{2}=0.2174, L_{3}=0, L_{4}=-12.9513
$$

Thus, the maximal Lyapunov exponent (MLE) of the system (1) is obtained as $L_{1}=$ 0.3684 . The system (1) is dissipative, because $\sum_{i=1}^{4} L_{i}=-12.3655<0$. Also, the KaplanYorke dimension of the 4-D hyperchaotic Lorenz system (1) is found as

$$
D_{K Y}=3+\frac{L_{1}+L_{2}+L_{3}}{\left|L_{4}\right|}=3.0452
$$


For numerical simulations, we take the initial state of the hyperchaotic system (1) as $x_{1}(0)=1.2, x_{2}(0)=0.8, x_{3}(0)=1.6$ and $x_{4}(0)=0.7$.

Figs. 1-2 depict the 3-D phase portraits of the 4-D hyperchaotic system (1) in $\left(x_{1}, x_{2}, x_{3}\right)$, and $\left(x_{2}, x_{3}, x_{4}\right)$ spaces, respectively.

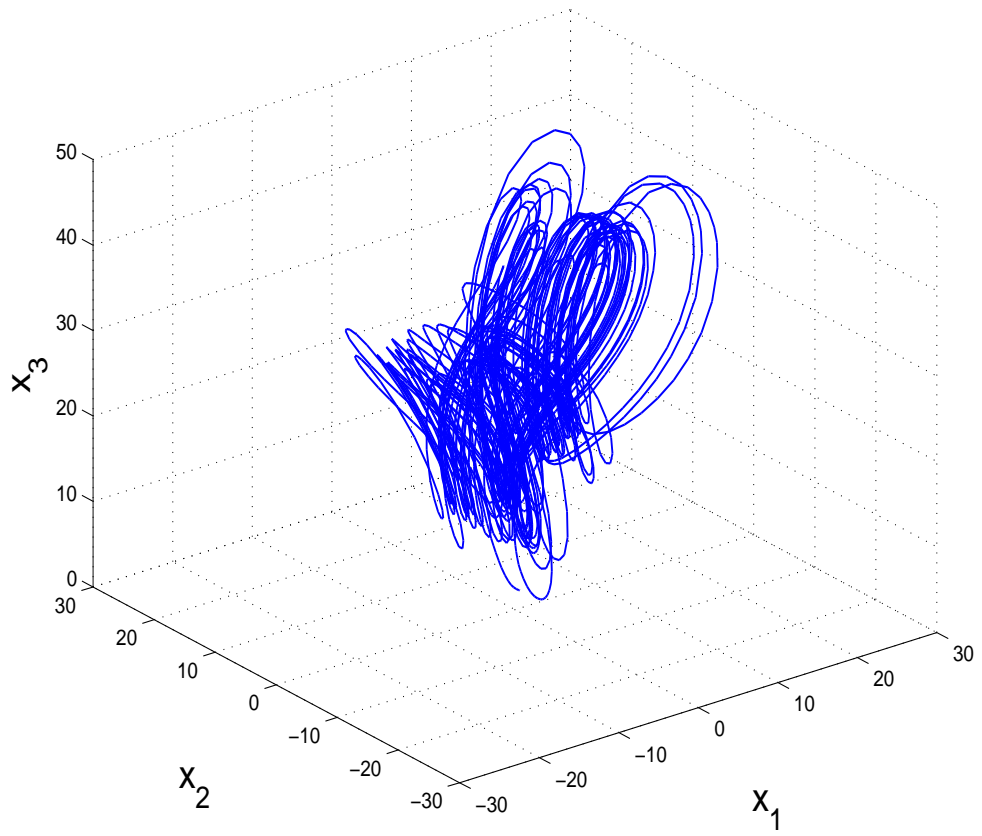

Figure 1: 3-D projection of the 4-D hyperchaotic Lorenz system on $\left(x_{1}, x_{2}, x_{3}\right)$ space.

In this research work, we derive a twelve-term novel 5-D hyperchaotic system with three quadratic nonlinearities by adding a feedback control to the ten-term 4-D hyperchaotic Lorenz system (1) as follows:

$$
\left\{\begin{array}{l}
\dot{x}_{1}=a\left(x_{2}-x_{1}\right)+x_{4}+x_{5} \\
\dot{x}_{2}=c x_{1}-x_{1} x_{3}-x_{2} \\
\dot{x}_{3}=x_{1} x_{2}-b x_{3} \\
\dot{x}_{4}=-x_{1} x_{3}+p x_{4} \\
\dot{x}_{5}=q x_{1}
\end{array}\right.
$$

where $x_{1}, x_{2}, x_{3}, x_{4}, x_{5}$ are the system parameters and $a, b, c, p, q$ are positive, constant, parameters.

The 5-D system (5) is hyperchaotic when the parameter values are taken as

$$
a=10, b=8 / 3, c=28, \quad p=1.3, q=2.5
$$




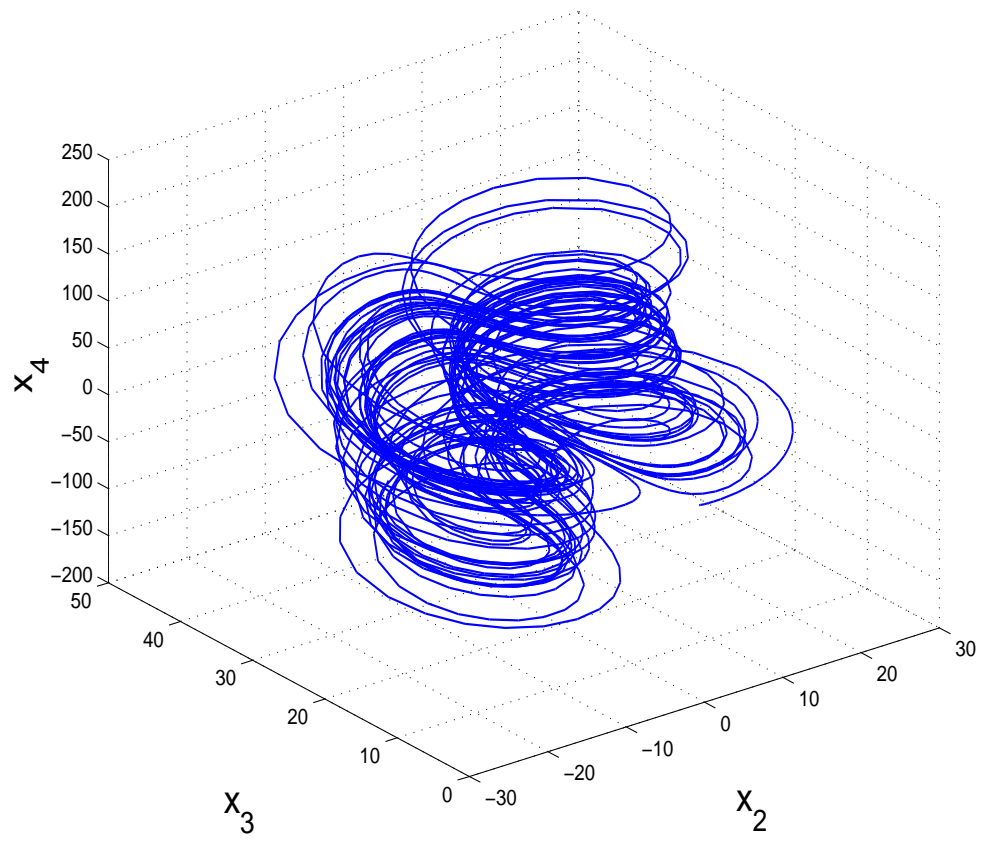

Figure 2: 3-D projection of the 4-D hyperchaotic Lorenz system on $\left(x_{2}, x_{3}, x_{4}\right)$ space.

Also, for these parameter values, the Lyapunov exponents of the 5-D novel hyperchaotic system (5) are calculated as

$$
L_{1}=0.4195, L_{2}=0.2430, L_{3}=0.0145, L_{4}=0, L_{5}=-13.0405
$$

Thus, the 5-D novel hyperchaotic system (5) has three positive Lyapunov exponents. Also, the maximal Lyapunov exponent (MLE) of the system (5) is obtained as $L_{1}=0.4195$, which is greater than the maximal Lyapunov exponent (MLE) of the 4-D hyperchaotic Lorenz system (1). Since the sum of the Lyapunov exponents of the 5-D novel hyperchaotic system (5) is negative, it follows that the system is dissipative.

Also, the Kaplan-Yorke dimension of the 5-D novel hyperchaotic Lorenz system (5) is found as

$$
D_{K Y}=4+\frac{L_{1}+L_{2}+L_{3}+L_{4}}{\left|L_{5}\right|}=4.0159
$$

Since the 5-D hyperchaotic system (5) has three positive Lyapunov exponents, it has a very complex dynamics and its trajectories can expand in three directions simultaneously.

For numerical simulations, we take the initial state of the 5-D novel hyperchaotic system $(5)$ as $x_{1}(0)=1.2, x_{2}(0)=0.8, x_{3}(0)=1.6, x_{4}(0)=0.7$ and $x_{5}(0)=2.3$.

Figs. 3-6 depict the 3-D phase portraits of the 5-D novel hyperchaotic system (5) in $\left(x_{1}, x_{2}, x_{3}\right),\left(x_{2}, x_{3}, x_{4}\right),\left(x_{1}, x_{2}, x_{5}\right)$ and $\left(x_{3}, x_{4}, x_{5}\right)$ spaces, respectively. 


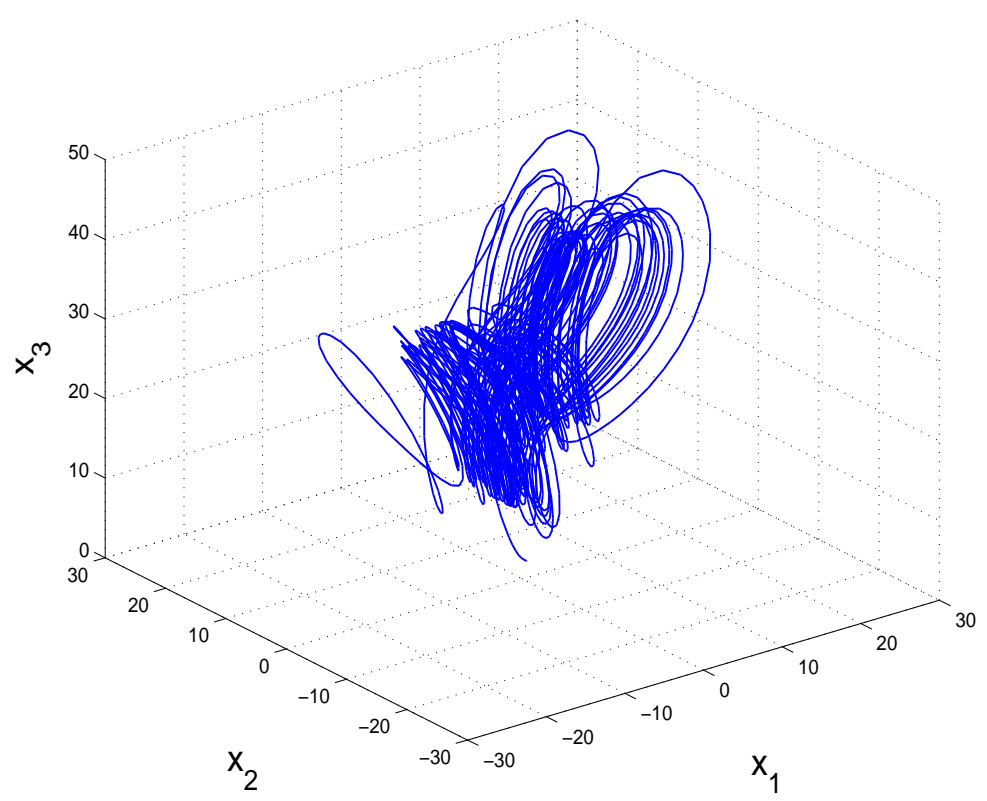

Figure 3: 3-D projection of the 5-D novel hyperchaotic Lorenz system on $\left(x_{1}, x_{2}, x_{3}\right)$ space.

\section{Analysis of the 5-D novel hyperchaotic Lorenz system}

\subsection{Dissipativity}

In vector notation, the novel 5-D hyperchaotic Lorenz system (5) can be expressed as

$$
\dot{\boldsymbol{x}}=f(\boldsymbol{x})=\left[\begin{array}{l}
f_{1}\left(x_{1}, x_{2}, x_{3}, x_{4}, x_{5}\right) \\
f_{2}\left(x_{1}, x_{2}, x_{3}, x_{4}, x_{5}\right) \\
f_{3}\left(x_{1}, x_{2}, x_{3}, x_{4}, x_{5}\right) \\
f_{4}\left(x_{1}, x_{2}, x_{3}, x_{4}, x_{5}\right) \\
f_{5}\left(x_{1}, x_{2}, x_{3}, x_{4}, x_{5}\right)
\end{array}\right],
$$

where

$$
\left\{\begin{array}{l}
f_{1}\left(x_{1}, x_{2}, x_{3}, x_{4}, x_{5}\right)=a\left(x_{2}-x_{1}\right)+x_{4}+x_{5} \\
f_{2}\left(x_{1}, x_{2}, x_{3}, x_{4}, x_{5}\right)=c x_{1}-x_{1} x_{3}-x_{2} \\
f_{3}\left(x_{1}, x_{2}, x_{3}, x_{4}, x_{5}\right)=x_{1} x_{2}-b x_{3} \\
f_{4}\left(x_{1}, x_{2}, x_{3}, x_{4}, x_{5}\right)=-x_{1} x_{3}+p x_{4} \\
f_{5}\left(x_{1}, x_{2}, x_{3}, x_{4}, x_{5}\right)=q x_{1}
\end{array}\right.
$$




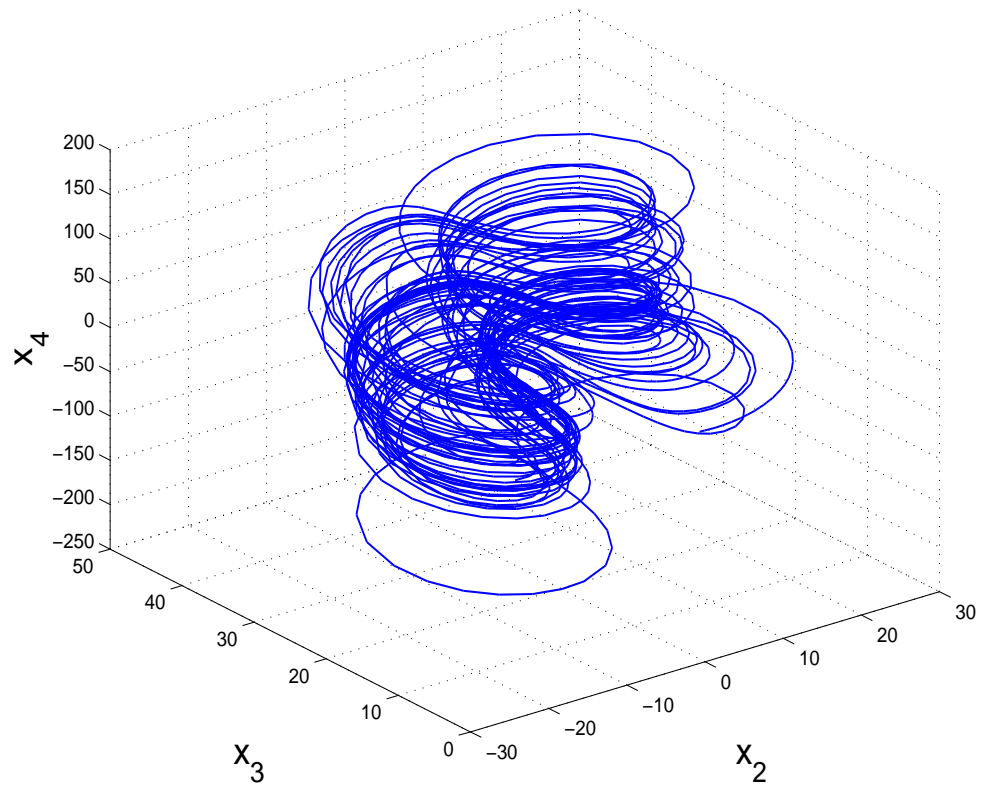

Figure 4: 3-D projection of the 5-D novel hyperchaotic Lorenz system on $\left(x_{2}, x_{3}, x_{4}\right)$ space.

Let $\Omega$ be any region in $\Re^{5}$ with a smooth boundary and also, $\Omega(t)=\Phi_{t}(\Omega)$, where $\Phi_{t}$ is the flow of $f$. Furthermore, let $V(t)$ denote the volume of $\Omega(t)$.

By Liouville's theorem, we know that

$$
\dot{V}(t)=\int_{\Omega(t)}(\nabla \cdot f) d x_{1} d x_{2} d x_{3} d x_{4} d x_{5}
$$

The divergence of the novel 5-D system (5) is found as:

$$
\nabla \cdot f=\frac{\partial f_{1}}{\partial x_{1}}+\frac{\partial f_{2}}{\partial x_{2}}+\frac{\partial f_{3}}{\partial x_{3}}+\frac{\partial f_{1}}{\partial x_{4}}+\frac{\partial f_{1}}{\partial x_{5}}=-a-1-b+p=-\mu
$$

where $\mu$ is defined as

$$
\mu=a+1+b-p
$$

For the choice of parameter values given in (6), we find that $\mu=12.3667>0$.

Inserting the value of $\nabla \cdot f$ from (12) into (11), we get

$$
\dot{V}(t)=\int_{\Omega(t)}(-\mu) d x_{1} d x_{2} d x_{3} d x_{4} d x_{5}=-\mu V(t)
$$

Integrating the first order linear differential equation (14), we get

$$
V(t)=\exp (-\mu t) V(0)
$$




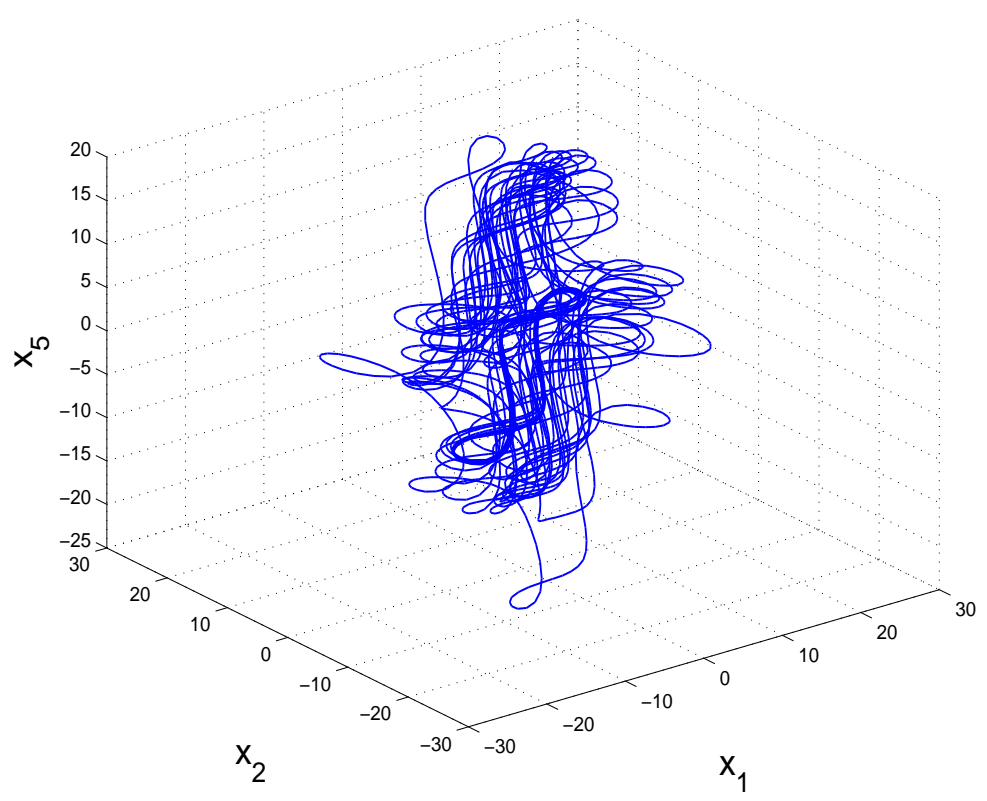

Figure 5: 3-D projection of the 5-D novel hyperchaotic Lorenz system on $\left(x_{1}, x_{2}, x_{5}\right)$ space.

Since $\mu>0$, it follows from (15) that $V(t) \rightarrow 0$ exponentially as $t \rightarrow \infty$. This shows that the 5-D novel hyperchaotic Lorenz system (5) is dissipative. Hence, the system limit sets are ultimately confined into a specific limit set of zero volume, and the asymptotic motion of the 5-D novel hyperchaotic Lorenz system (5) settles onto a strange attractor of the system.

\subsection{Equilibrium Points}

The equilibrium points of the 5-D novel hyperchaotic system (5) are obtained by solving the equations

$$
\left.\begin{array}{ll}
f_{1}\left(x_{1}, x_{2}, x_{3}, x_{4}, x_{5}\right)=a\left(x_{2}-x_{1}\right)+x_{4}+x_{5} & =0 \\
f_{2}\left(x_{1}, x_{2}, x_{3}, x_{4}, x_{5}\right)=c x_{1}-x_{1} x_{3}-x_{2} & =0 \\
f_{3}\left(x_{1}, x_{2}, x_{3}, x_{4}, x_{5}\right)=x_{1} x_{2}-b x_{3} & =0 \\
f_{4}\left(x_{1}, x_{2}, x_{3}, x_{4}, x_{5}\right)=-x_{1} x_{3}+p x_{4} & =0 \\
f_{5}\left(x_{1}, x_{2}, x_{3}, x_{4}, x_{5}\right)=q x_{1} & =0
\end{array}\right\}
$$

We take the parameter values as in the equation (6).

Since $\boldsymbol{x}^{\star}=\mathbf{0}$ is the unique solution of the system of equations (16), it is immediate that $\boldsymbol{x}^{\star}=\mathbf{0}$ is the unique equilibrium of the 5-D novel hyperchaotic Lorenz system (5). 


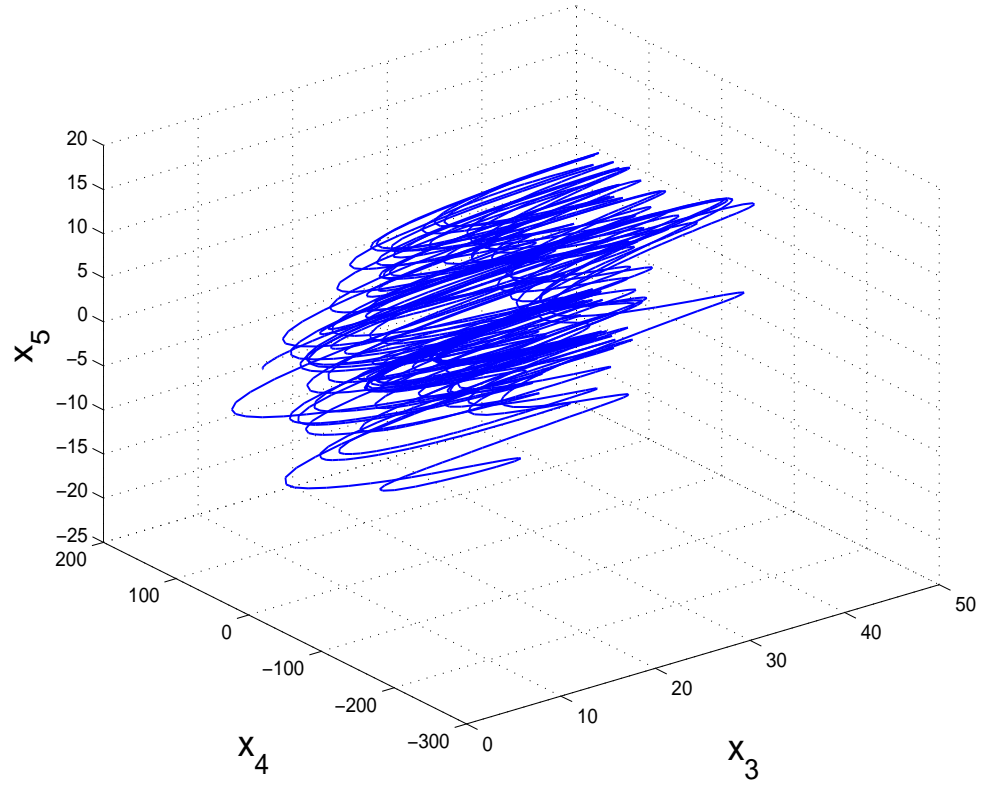

Figure 6: 3-D projection of the 5-D novel hyperchaotic Lorenz system on $\left(x_{3}, x_{4}, x_{5}\right)$ space.

The Jacobian matrix of the 5-D hyperchaotic Lorenz system (5) at the equilibrium point $\boldsymbol{x}^{\star}=\mathbf{0}$ is given by

$$
J\left(\boldsymbol{x}^{\star}\right)=\left[\begin{array}{ccccc}
-a & a & 0 & 1 & 1 \\
c & -1 & 0 & 0 & 0 \\
0 & 0 & -b & 0 & 0 \\
0 & 0 & 0 & p & 0 \\
q & 0 & 0 & 0 & 0
\end{array}\right]=\left[\begin{array}{ccccc}
-10 & 10 & 0 & 1 & 1 \\
28 & -1 & 0 & 0 & 0 \\
0 & 0 & -8 / 3 & 0 & 0 \\
0 & 0 & 0 & 1.3 & 0 \\
2.5 & 0 & 0 & 0 & 0
\end{array}\right]
$$

The eigenvalues of the matrix $J\left(\boldsymbol{x}^{\star}\right)$ are numerically obtained as

$$
\lambda_{1}=1.3, \lambda_{2}=11.9057, \lambda_{3}=-0.0092, \lambda_{4}=-2.6667, \lambda_{5}=-22.8966
$$

Thus, the equilibrium point $\boldsymbol{x}^{\star}=\mathbf{0}$ is a saddle-point, which is unstable. 


\subsection{Rotation symmetry about the $x_{3}$-axis}

We define a new set of coordinates as

$$
\begin{aligned}
& \xi_{1}=-x_{1} \\
& \xi_{2}=-x_{2} \\
& \xi_{3}=x_{3} \\
& \xi_{4}=-x_{4} \\
& \xi_{5}=-x_{5}
\end{aligned}
$$

We find that

$$
\begin{aligned}
& \dot{\xi}_{1}=-a\left(x_{2}-x_{1}\right)-x_{4}-x_{5}=a\left(\xi_{2}-\xi_{1}\right)+\xi_{4}+\xi_{5} \\
& \dot{\xi}_{2}=x_{1} x_{3}-c x_{1}+x_{2}=-\xi_{1} \xi_{3}+c \xi_{1}-\xi_{2} \\
& \dot{\xi}_{3}=x_{1} x_{2}-b x_{3}=\xi_{1} \xi_{2}-b \xi_{3} \\
& \dot{\xi}_{4}=x_{1} x_{3}-p x_{4}=-\xi_{1} \xi_{3}+p \xi_{4} \\
& \dot{\xi}_{5}=-q x_{1}=q \xi_{1}
\end{aligned}
$$

This shows that the 5-D novel hyperchaotic Lorenz system (5) is invariant under the change of coordinates

$$
\left(x_{1}, x_{2}, x_{3}, x_{4}, x_{5}\right) \mapsto\left(-x_{1},-x_{2}, x_{3},-x_{4},-x_{5}\right)
$$

Since the transformation (21) persists for all values of the system parameters, it follows that the 5-D novel hyperchaotic Lorenz system (5) has rotation symmetry about the $x_{3}$-axis and that any non-trivial trajectory must have a twin trajectory.

\subsection{Invariance}

It is easy to see that the $x_{3}$-axis and $x_{4}$-axis are invariant under the flow of the 5-D novel hyperchaotic Lorenz system (5). The invariant motion along the $x_{3}$-axis is characterized by the scalar dynamics

$$
\dot{x}_{3}=-b x_{3}, \quad(b>0)
$$

which is globally exponentially stable. The invariant motion along the $x_{4}$-axis is characterized by the scalar dynamics

$$
\dot{x}_{4}=p x_{4}, \quad(p>0)
$$

which is unstable. 


\subsection{Lyapunov exponents and Kaplan-Yorke dimension}

For the parameter values given in the equation (6), the Lyapunov exponents of the 5-D novel hyperchaotic Lorenz system (5) are calculated as

$$
L_{1}=0.4195, L_{2}=0.2430, L_{3}=0.0145, L_{4}=0, L_{5}=-13.0405
$$

Thus, the 5-D novel hyperchaotic Lorenz system (5) has three positive Lyapunov exponents. Also, the maximal Lyapunov exponent (MLE) of the system (5) is obtained as $L_{1}=0.4195$.

Also, the Kaplan-Yorke dimension of the novel hyperchaotic system (5) is obtained as

$$
D_{K Y}=4+\frac{L_{1}+L_{2}+L_{3}+L_{4}}{\left|L_{5}\right|}=4.0159
$$

which is fractional.

Since the 5-D hyperchaotic Lorenz system (5) has three positive Lyapunov exponents, it has a very complex dynamics and the system trajectories can expand in three different directions.

\section{Adaptive control of the 5-D novel hyperchaotic Lorenz system with unknown parameters}

In this section, we use adaptive control method to derive an adaptive feedback control law for globally stabilizing the 5-D novel hyperchaotic Lorenz system with unknown parameters.

Thus, we consider the 5-D novel hyperchaotic Lorenz system given by

$$
\left\{\begin{aligned}
\dot{x}_{1} & =a\left(x_{2}-x_{1}\right)+x_{4}+x_{5}+u_{1} \\
\dot{x}_{2} & =c x_{1}-x_{1} x_{3}-x_{2}+u_{2} \\
\dot{x}_{3} & =x_{1} x_{2}-b x_{3}+u_{3} \\
\dot{x}_{4} & =-x_{1} x_{3}+p x_{4}+u_{4} \\
\dot{x}_{5} & =q x_{1}+u_{5}
\end{aligned}\right.
$$

In (26), $x_{i},(i=1, \ldots, 5)$ are the states and $u_{i},(i=1, \ldots, 5)$ are the adaptive controls to be determined using estimates $\hat{a}(t), \hat{b}(t), \hat{c}(t), \hat{p}(t), \hat{q}(t)$ for the unknown parameters $a, b, c, p, q$, respectively. 
We consider the adaptive feedback control law

$$
\left\{\begin{array}{l}
u_{1}=-\hat{a}(t)\left(x_{2}-x_{1}\right)-x_{4}-x_{5}-k_{1} x_{1} \\
u_{2}=-\hat{c}(t) x_{1}+x_{1} x_{3}+x_{2}-k_{2} x_{2} \\
u_{3}=-x_{1} x_{2}+\hat{b}(t) x_{3}-k_{3} x_{3} \\
u_{4}=x_{1} x_{3}-\hat{p}(t) x_{4}-k_{4} x_{4} \\
u_{5}=-\hat{q}(t) x_{1}-k_{5} x_{5}
\end{array}\right.
$$

where $k_{i},(i=1, \ldots, 5)$ are positive gain constants.

Substituting (27) into (26), we get the closed-loop plant dynamics as

$$
\left\{\begin{aligned}
\dot{x}_{1} & =[a-\hat{a}(t)]\left(x_{2}-x_{1}\right)-k_{1} x_{1} \\
\dot{x}_{2} & =[c-\hat{c}(t)] x_{1}-k_{2} x_{2} \\
\dot{x}_{3} & =-[b-\hat{b}(t)] x_{3}-k_{3} x_{3} \\
\dot{x}_{4} & =[p-\hat{p}(t)] x_{4}-k_{4} x_{4} \\
\dot{x}_{5} & =[q-\hat{q}(t)] x_{1}-k_{5} x_{5}
\end{aligned}\right.
$$

The parameter estimation errors are defined as

$$
\left\{\begin{array}{l}
e_{a}(t)=a-\hat{a}(t) \\
e_{b}(t)=b-\hat{b}(t) \\
e_{c}(t)=c-\hat{c}(t) \\
e_{p}(t)=p-\hat{p}(t) \\
e_{q}(t)=q-\hat{q}(t)
\end{array}\right.
$$

In view of (29), we can simplify the plant dynamics (28) as

$$
\left\{\begin{array}{l}
\dot{x}_{1}=e_{a}\left(x_{2}-x_{1}\right)-k_{1} x_{1} \\
\dot{x}_{2}=e_{c} x_{1}-k_{2} x_{2} \\
\dot{x}_{3}=-e_{b} x_{3}-k_{3} x_{3} \\
\dot{x}_{4}=e_{p} x_{4}-k_{4} x_{4} \\
\dot{x}_{5}=e_{q} x_{1}-k_{5} x_{5}
\end{array}\right.
$$


Differentiating (29) with respect to $t$, we obtain

$$
\left\{\begin{array}{l}
\dot{e}_{a}(t)=-\dot{\hat{a}}(t) \\
\dot{e}_{b}(t)=-\dot{\hat{b}}(t) \\
\dot{e}_{c}(t)=-\dot{\hat{c}}(t) \\
\dot{e}_{p}(t)=-\dot{\hat{p}}(t) \\
\dot{e}_{q}(t)=-\dot{\hat{q}}(t)
\end{array}\right.
$$

We use adaptive control theory to find an update law for the parameter estimates.

We consider the quadratic candidate Lyapunov function defined by

$$
V\left(\boldsymbol{x}, e_{a}, e_{b}, e_{c}, e_{p}, e_{q}\right)=\frac{1}{2} \sum_{i=1}^{5} x_{i}^{2}+\frac{1}{2}\left(e_{a}^{2}+e_{b}^{2}+e_{c}^{2}+e_{p}^{2}+e_{q}^{2}\right)
$$

Differentiating $V$ along the trajectories of (30) and (31), we obtain

$$
\begin{aligned}
\dot{V}= & -k_{1} x_{1}^{2}-k_{2} x_{2}^{2}-k_{3} x_{3}^{2}-k_{4} x_{4}^{2}-k_{5} x_{5}^{2} \\
& +e_{a}\left[x_{1}\left(x_{2}-x_{1}\right)-\dot{\hat{a}}\right]+e_{b}\left[-x_{3}^{2}-\dot{\hat{b}}\right]+e_{c}\left[x_{1} x_{2}-\dot{\hat{c}}\right] \\
& +e_{p}\left[x_{4}^{2}-\dot{\hat{p}}\right]+e_{q}\left[x_{1} x_{5}-\dot{\hat{q}}\right]
\end{aligned}
$$

In view of (33), we take the parameter update law as

$$
\left\{\begin{array}{l}
\dot{\hat{a}}(t)=x_{1}\left(x_{2}-x_{1}\right) \\
\dot{\hat{b}}(t)=-x_{3}^{2} \\
\dot{\hat{c}}(t)=x_{1} x_{2} \\
\dot{\hat{p}}(t)=x_{4}^{2} \\
\dot{\hat{q}}(t)=x_{1} x_{5}
\end{array}\right.
$$

Next, we state and prove the main result of this section.

Theorem 1 The novel 5-D hyperchaotic Lorenz system (26) with unknown system parameters is globally and exponentially stabilized for all initial conditions by the adaptive control law (27) and the parameter update law (34), where $k_{1}, k_{2}, k_{3}, k_{4}, k_{5}$ are positive gain constants.

Proof We prove this result by applying Lyapunov stability theory.

We consider the quadratic Lyapunov function defined by (32), which is clearly a positive definite function on $\Re^{10}$. 
By substituting the parameter update law (34) into (33), we obtain the time-derivative of $V$ as

$$
\dot{V}=-k_{1} x_{1}^{2}-k_{2} x_{2}^{2}-k_{3} x_{3}^{2}-k_{4} x_{4}^{2}-k_{5} x_{5}^{2}
$$

From (35), it is clear that $\dot{V}$ is a negative semi-definite function on $\Re^{10}$.

Thus, we can conclude that the state vector $\boldsymbol{x}(t)$ and the parameter estimation error are globally bounded, i.e.

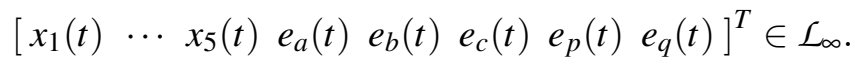

We define $k=\min \left\{k_{1}, k_{2}, k_{3}, k_{4}, k_{5}\right\}$.

Then it follows from (35) that

$$
\dot{V} \leqslant-k\|\boldsymbol{x}(t)\|^{2}
$$

Thus, we have

$$
k\|x(t)\|^{2} \leqslant-\dot{V}
$$

Integrating the inequality (37) from 0 to $t$, we get

$$
k \int_{0}^{t}\|\boldsymbol{x}(\tau)\|^{2} d \tau \leqslant V(0)-V(t)
$$

From (38), it follows that $x \in \mathcal{L}_{2}$.

Using (30), we can conclude that $\dot{\boldsymbol{x}} \in \mathcal{L}_{\infty}$.

Using Barbalat's lemma, we conclude that $\boldsymbol{x}(t) \rightarrow 0$ exponentially as $t \rightarrow \infty$ for all initial conditions $\boldsymbol{x}(0) \in \mathfrak{R}^{5}$.

This completes the proof.

For the numerical simulations, the classical fourth-order Runge-Kutta method with step size $h=10^{-8}$ is used to solve the systems (26) and (34), when the adaptive control law (27) is applied.

The parameter values of the novel 5-D hyperchaotic Lorenz system (26) are taken as in the hyperchaotic case, viz. $a=10, b=8 / 3, c=28, p=1.3$ and $q=2.5$. We take the positive gain constants as $k_{i}=5$ for $i=1, \ldots, 5$.

Furthermore, as initial conditions of the novel 5-D hyperchaotic Lorenz system (26), we take $x_{1}(0)=5.2, x_{2}(0)=3.8, x_{3}(0)=-11.2, x_{4}(0)=-4.5$ and $x_{5}(0)=3.5$.

Also, as initial conditions of the parameter estimates $\hat{a}(t), \hat{b}(t), \hat{c}(t), \hat{p}(t), \hat{q}(t)$, we take $\hat{a}(0)=4.2, \hat{b}(0)=6.8, \hat{c}(0)=-3.5, \hat{p}(0)=8.2$ and $\hat{q}(0)=7.4$.

In Fig. 7, the exponential convergence of the controlled states of the novel 5-D hyperchaotic Lorenz system (26) is depicted. 


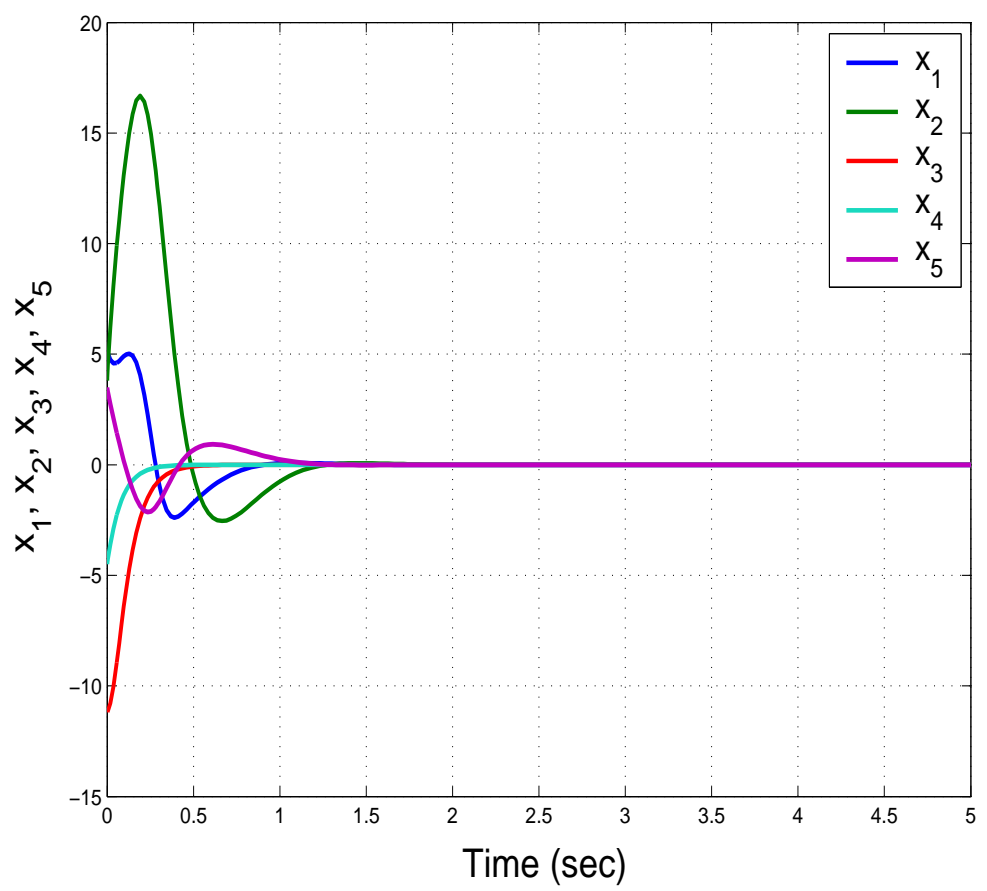

Figure 7: Time-history of the controlled states $x_{1}(t), x_{2}(t), x_{3}(t), x_{4}(t), x_{5}(t)$.

\section{Adaptive synchronization of the 5-D novel hyperchaotic Lorenz systems with unknown parameters}

In this section, we use adaptive control method to derive an adaptive feedback control law for globally synchronizing identical 5-D novel hyperchaotic Lorenz systems with unknown parameters.

As the master system, we consider the 5-D novel hyperchaotic Lorenz system given by

$$
\left\{\begin{array}{l}
\dot{x}_{1}=a\left(x_{2}-x_{1}\right)+x_{4}+x_{5} \\
\dot{x}_{2}=c x_{1}-x_{1} x_{3}-x_{2} \\
\dot{x}_{3}=x_{1} x_{2}-b x_{3} \\
\dot{x}_{4}=-x_{1} x_{3}+p x_{4} \\
\dot{x}_{5}=q x_{1}
\end{array}\right.
$$

In (39), $x_{1}, x_{2}, x_{3}, x_{4}, x_{5}$ are the states and $a, b, c, p, q$ are unknown system parameters. 
As the slave system, we consider the 5-D novel hyperchaotic Lorenz system given by

$$
\left\{\begin{array}{l}
\dot{y}_{1}=a\left(y_{2}-y_{1}\right)+y_{4}+y_{5}+u_{1} \\
\dot{y}_{2}=c y_{1}-y_{1} y_{3}-y_{2}+u_{2} \\
\dot{y}_{3}=y_{1} y_{2}-b y_{3}+u_{3} \\
\dot{y}_{4}=-y_{1} y_{3}+p y_{4}+u_{4} \\
\dot{y}_{5}=q y_{1}+u_{5}
\end{array}\right.
$$

In (40), $y_{1}, y_{2}, y_{3}, y_{4}, y_{5}$ are the states and $u_{1}, u_{2}, u_{3}, u_{4}, u_{5}$ are the adaptive controls to be determined using estimates $\hat{a}(t), \hat{b}(t), \hat{c}(t), \hat{p}(t), \hat{q}(t)$ for the unknown parameters $a, b, c, p, q$, respectively.

The synchronization error between the novel 5-D hyperchaotic systems (39) and (40) is defined by

$$
e_{i}=y_{i}-x_{i}, \quad(i=1,2, \ldots, 5)
$$

Then the synchronization error dynamics is obtained as

$$
\left\{\begin{array}{l}
\dot{e}_{1}=a\left(e_{2}-e_{1}\right)+e_{4}+e_{5}+u_{1} \\
\dot{e}_{2}=c e_{1}-y_{1} y_{3}+x_{1} x_{3}-e_{2}+u_{2} \\
\dot{e}_{3}=y_{1} y_{2}-x_{1} x_{2}-b e_{3}+u_{3} \\
\dot{e}_{4}=-y_{1} y_{3}+x_{1} x_{3}+p e_{4}+u_{4} \\
\dot{e}_{5}=q e_{1}+u_{5}
\end{array}\right.
$$

We consider the adaptive feedback control law

$$
\left\{\begin{array}{l}
u_{1}=-\hat{a}(t)\left(e_{2}-e_{1}\right)-e_{4}-e_{5}-k_{1} e_{1} \\
u_{2}=-\hat{c}(t) e_{1}+y_{1} y_{3}-x_{1} x_{3}+e_{2}-k_{2} e_{2} \\
u_{3}=-y_{1} y_{2}+x_{1} x_{2}+\hat{b}(t) e_{3}-k_{3} e_{3} \\
u_{4}=y_{1} y_{3}-x_{1} x_{3}-\hat{p}(t) e_{4}-k_{4} e_{4} \\
u_{5}=-\hat{q}(t) e_{1}-k_{5} e_{5}
\end{array}\right.
$$

where $k_{i},(i=1, \ldots, 5)$ are positive gain constants. 
Substituting (43) into (42), we get the closed-loop error dynamics as

$$
\left\{\begin{array}{l}
\dot{e}_{1}=[a-\hat{a}(t)]\left(e_{2}-e_{1}\right)-k_{1} e_{1} \\
\dot{e}_{2}=[c-\hat{c}(t)] e_{1}-k_{2} e_{2} \\
\dot{e}_{3}=-[b-\hat{b}(t)] e_{3}-k_{3} e_{3} \\
\dot{e}_{4}=[p-\hat{p}(t)] e_{4}-k_{4} e_{4} \\
\dot{e}_{5}=[q-\hat{q}(t)] e_{1}-k_{5} e_{5}
\end{array}\right.
$$

The parameter estimation errors are defined as

$$
\left\{\begin{array}{l}
e_{a}(t)=a-\hat{a}(t) \\
e_{b}(t)=b-\hat{b}(t) \\
e_{c}(t)=c-\hat{c}(t) \\
e_{p}(t)=p-\hat{p}(t) \\
e_{q}(t)=q-\hat{q}(t)
\end{array}\right.
$$

In view of (45), we can simplify the plant dynamics (44) as

$$
\left\{\begin{array}{l}
\dot{e}_{1}=e_{a}\left(e_{2}-e_{1}\right)-k_{1} e_{1} \\
\dot{e}_{2}=e_{c} e_{1}-k_{2} e_{2} \\
\dot{e}_{3}=-e_{b} e_{3}-k_{3} e_{3} \\
\dot{e}_{4}=e_{p} e_{4}-k_{4} e_{4} \\
\dot{e}_{5}=e_{q} e_{1}-k_{5} e_{5}
\end{array}\right.
$$

Differentiating (45) with respect to $t$, we obtain

$$
\left\{\begin{array}{l}
\dot{e}_{a}(t)=-\dot{\hat{a}}(t) \\
\dot{e}_{b}(t)=-\dot{\hat{b}}(t) \\
\dot{e}_{c}(t)=-\dot{\hat{c}}(t) \\
\dot{e}_{p}(t)=-\dot{\hat{p}}(t) \\
\dot{e}_{q}(t)=-\dot{\hat{q}}(t)
\end{array}\right.
$$

We use adaptive control theory to find an update law for the parameter estimates. We consider the quadratic candidate Lyapunov function defined by

$$
V\left(\boldsymbol{e}, e_{a}, e_{b}, e_{c}, e_{p}, e_{q}\right)=\frac{1}{2} \sum_{i=1}^{5} e_{i}^{2}+\frac{1}{2}\left(e_{a}^{2}+e_{b}^{2}+e_{c}^{2}+e_{p}^{2}+e_{q}^{2}\right)
$$


Differentiating $V$ along the trajectories of (46) and (47), we obtain

$$
\begin{aligned}
\dot{V}= & -k_{1} e_{1}^{2}-k_{2} e_{2}^{2}-k_{3} e_{3}^{2}-k_{4} e_{4}^{2}-k_{5} e_{5}^{2} \\
& +e_{a}\left[e_{1}\left(e_{2}-e_{1}\right)-\dot{\hat{a}}\right]+e_{b}\left[-e_{3}^{2}-\dot{\hat{b}}\right] \\
& +e_{c}\left[e_{1} e_{2}-\dot{\hat{c}}\right]+e_{p}\left[e_{4}^{2}-\dot{\hat{p}}\right]+e_{q}\left[e_{1} e_{5}-\dot{\hat{q}}\right]
\end{aligned}
$$

In view of (49), we take the parameter update law as

$$
\left\{\begin{array}{l}
\dot{\hat{a}}(t)=e_{1}\left(e_{2}-e_{1}\right) \\
\dot{\hat{b}}(t)=-e_{3}^{2} \\
\dot{\hat{c}}(t)=e_{1} e_{2} \\
\dot{\hat{p}}(t)=e_{4}^{2} \\
\dot{\hat{q}}(t)=e_{1} e_{5}
\end{array}\right.
$$

Next, we state and prove the main result of this section.

Theorem 2 The novel 5-D hyperchaotic Lorenz systems (39) and (40) with unknown system parameters are globally and exponentially synchronized for all initial conditions by the adaptive control law (43) and the parameter update law (50), where $k_{1}, k_{2}, k_{3}, k_{4}, k_{5}$ are positive gain constants.

Proof We prove this result by applying Lyapunov stability theory.

We consider the quadratic Lyapunov function defined by (48), which is clearly a positive definite function on $\Re^{10}$.

By substituting the parameter update law (50) into (49), we obtain the time-derivative of $V$ as

$$
\dot{V}=-k_{1} e_{1}^{2}-k_{2} e_{2}^{2}-k_{3} e_{3}^{2}-k_{4} e_{4}^{2}-k_{5} e_{5}^{2}
$$

From (51), it is clear that $\dot{V}$ is a negative semi-definite function on $\Re^{10}$.

Thus, we can conclude that the state vector $\boldsymbol{e}(t)$ and the parameter estimation error are globally bounded, i.e.

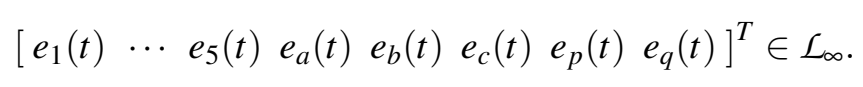

We define $k=\min \left\{k_{1}, k_{2}, k_{3}, k_{4}, k_{5}\right\}$.

Then it follows from (51) that

$$
\dot{V} \leqslant-k\|\boldsymbol{e}(t)\|^{2}
$$

Thus, we have

$$
k\|\boldsymbol{e}(t)\|^{2} \leqslant-\dot{V}
$$


Integrating the inequality (53) from 0 to $t$, we get

$$
k \int_{0}^{t}\|\boldsymbol{e}(\tau)\|^{2} d \tau \leqslant V(0)-V(t)
$$

From (54), it follows that $e \in \mathcal{L}_{2}$.

Using (46), we can conclude that $\dot{e} \in \mathcal{L}_{\infty}$.

Using Barbalat's lemma, we conclude that $\boldsymbol{e}(t) \rightarrow 0$ exponentially as $t \rightarrow \infty$ for all initial conditions $\boldsymbol{e}(0) \in \mathfrak{R}^{5}$.

This completes the proof.

For the numerical simulations, the classical fourth-order Runge-Kutta method with step size $h=10^{-8}$ is used to solve the systems (39), (40) and (50), when the adaptive control law (43) is applied.

The parameter values of the novel 5-D hyperchaotic systems are taken as in the hyperchaotic case, viz. $a=10, b=8 / 3, c=28, p=1.3$ and $q=2.5$. We take the positive gain constants as $k_{i}=5$ for $i=1, \ldots, 5$.

Furthermore, as initial conditions of the master system (39), we take

$$
x_{1}(0)=3.1, x_{2}(0)=-5.8, x_{3}(0)=7.3, x_{4}(0)=9.1, x_{5}(0)=-2.6
$$

As initial conditions of the slave system (40), we take

$$
y_{1}(0)=-8.4, \quad y_{2}(0)=3.5, \quad y_{3}(0)=4.2, \quad y_{4}(0)=-5.4, \quad y_{5}(0)=10.3
$$

Also, as initial conditions of the parameter estimates, we take

$$
\hat{a}(0)=3.1, \hat{b}(0)=12.4, \hat{c}(0)=4.7, \hat{p}(0)=-5.8, \hat{q}(0)=3.2
$$

Figs. 8-12 describe the complete synchronization of the 5-D novel hyperchaotic Lorenz systems (39) and (40), while Fig. 13 describes the time-history of the synchronization errors $e_{1}, e_{2}, e_{3}, e_{4}, e_{5}$. 


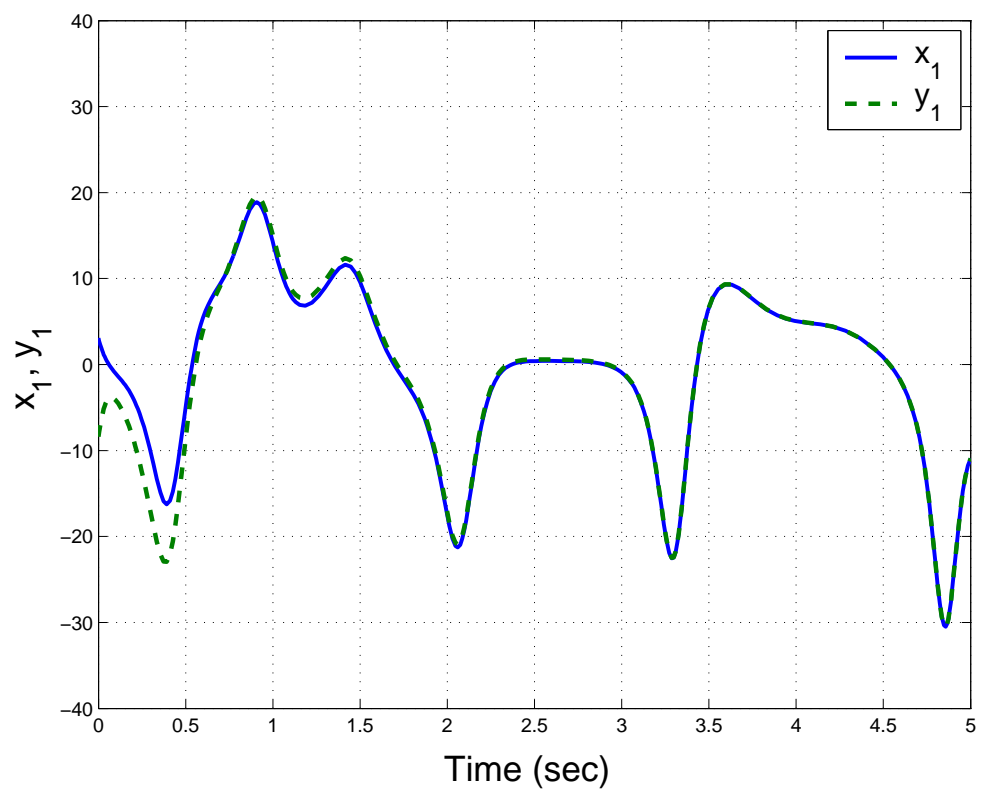

Figure 8: Synchronization of the states $x_{1}$ and $y_{1}$ of the 5-D novel hyperchaotic Lorenz systems.

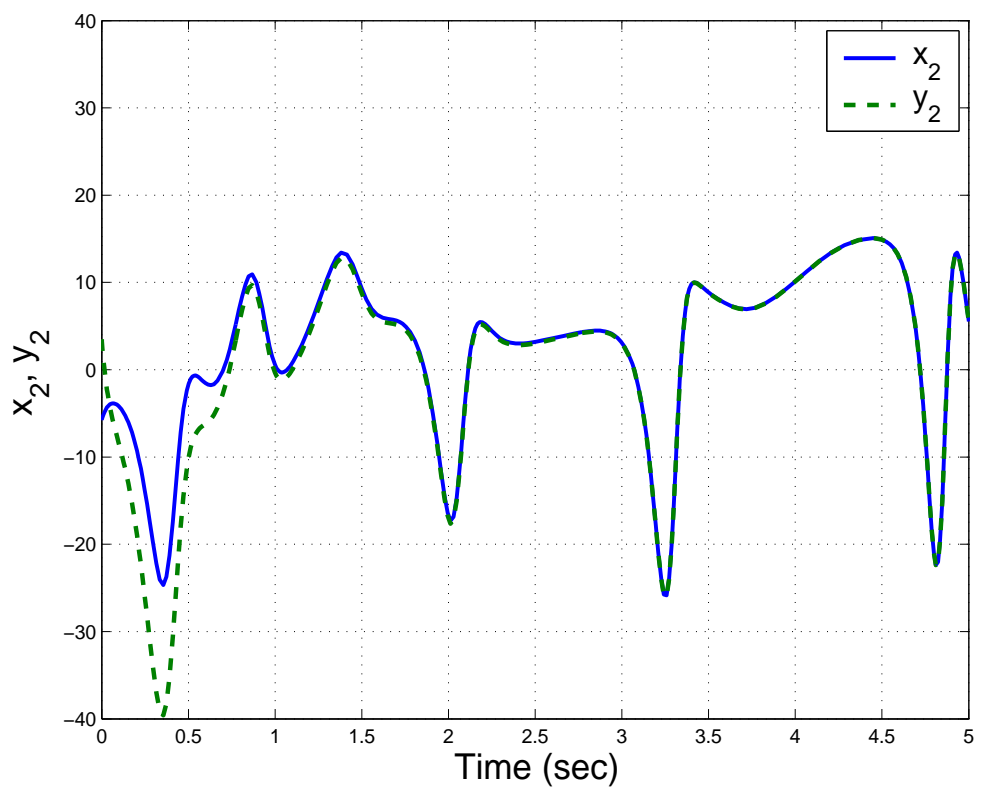

Figure 9: Synchronization of the states $x_{2}$ and $y_{2}$ of the 5-D novel hyperchaotic Lorenz systems. 


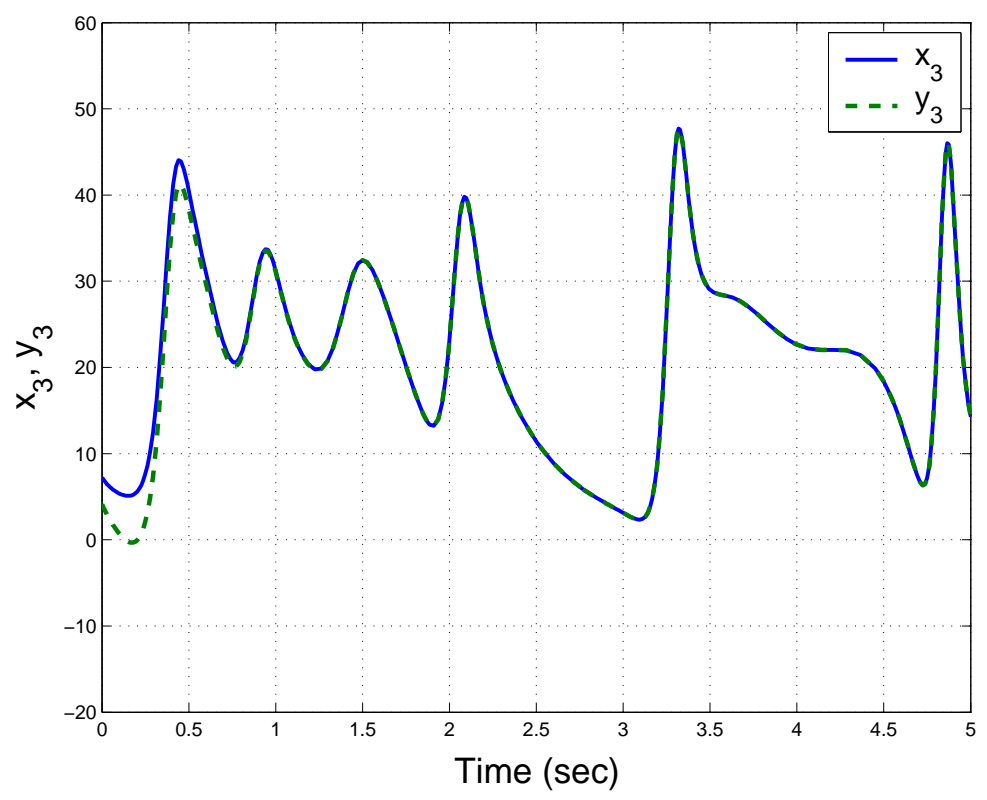

Figure 10: Synchronization of the states $x_{3}$ and $y_{3}$ of the 5-D novel hyperchaotic Lorenz systems.

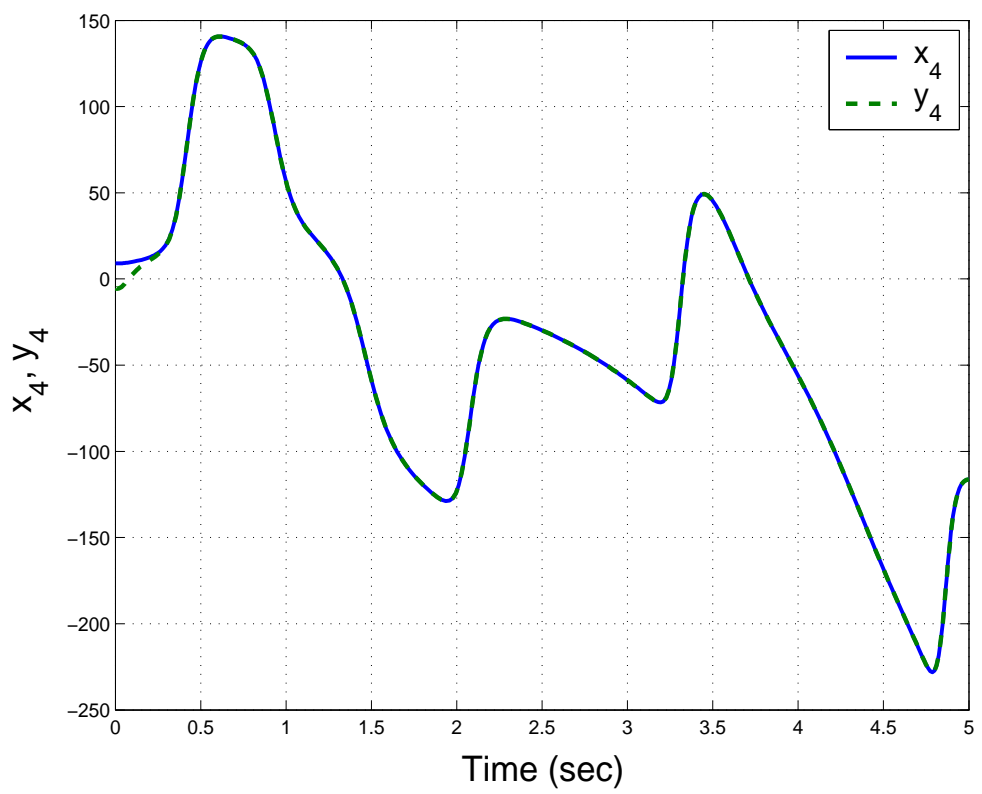

Figure 11: Synchronization of the states $x_{4}$ and $y_{4}$ of the 5-D novel hyperchaotic Lorenz systems. 


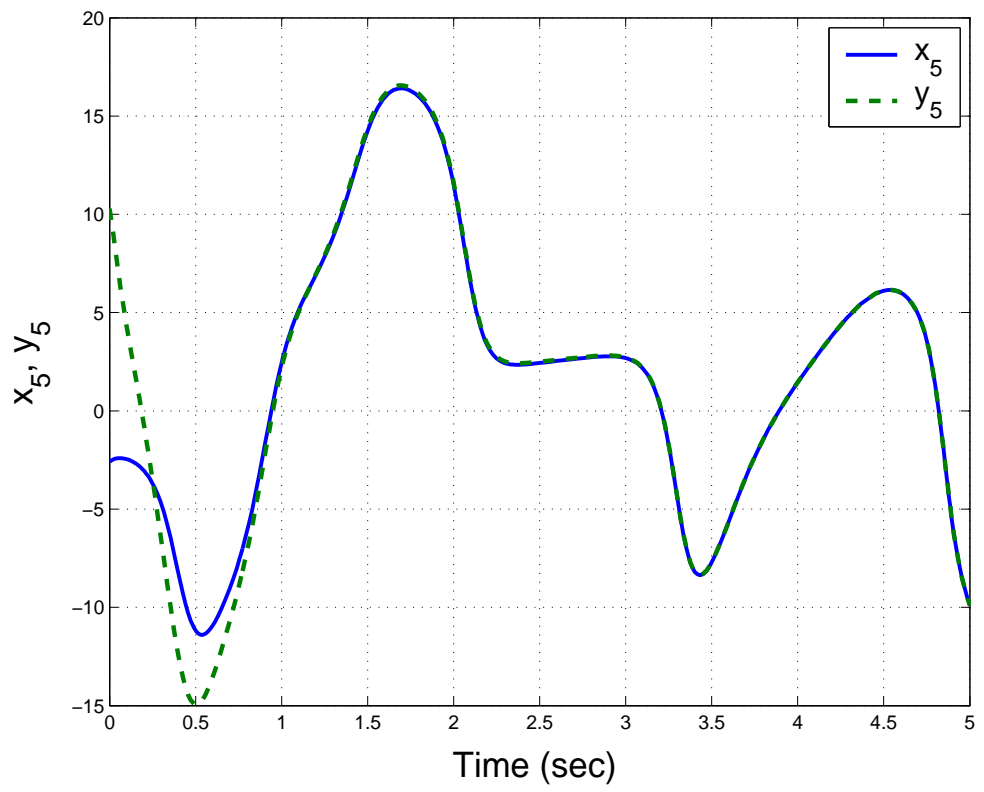

Figure 12: Synchronization of the states $x_{5}$ and $y_{5}$ of the 5-D novel hyperchaotic Lorenz systems.

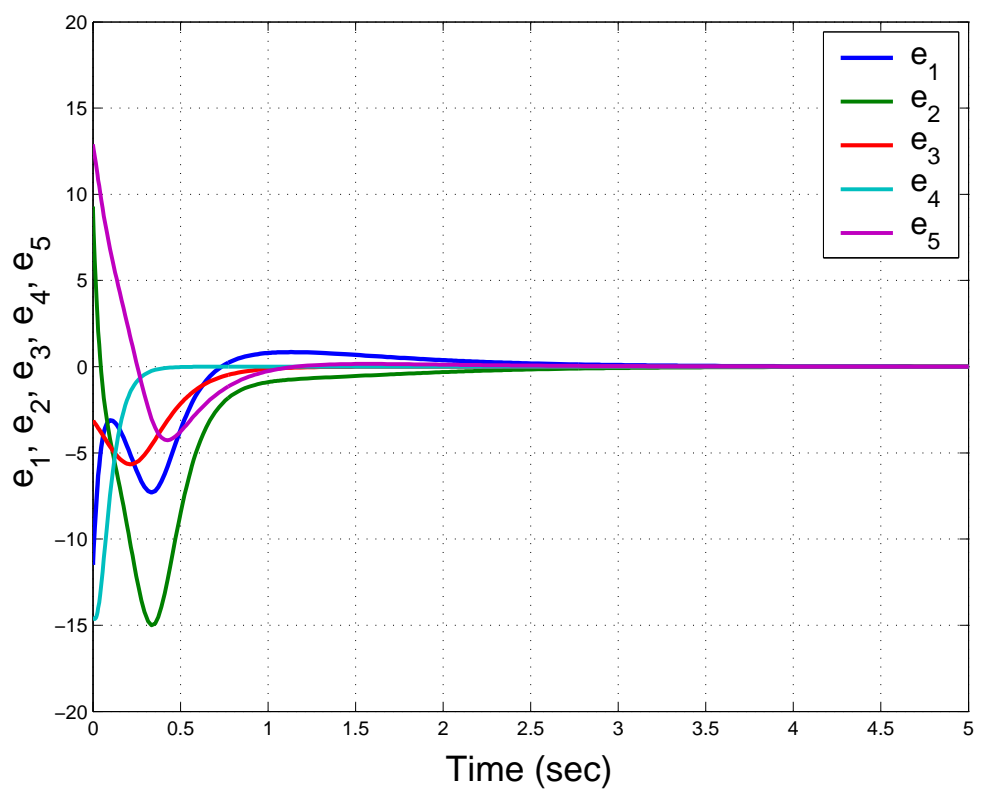

Figure 13: Time-history of the synchronization errors $e_{1}, e_{2}, e_{3}, e_{4}, e_{5}$. 


\section{Circuit realization of novel 5-D hyperchaotic Lorenz system}

In order to illustrate the correction and feasibility of novel hyperchaotic system (5), an electronic circuit modeling new system (5) is designed. Due to the fact that the electronic circuit is designed following an approach based in operational amplifiers [19, 29, 31], the state variables of system (5) are scaled down to obtain attractors in the dynamical range of operational amplifiers. As a result, the new hyperchaotic system (5) can be rewritten as

$$
\left\{\begin{array}{l}
\dot{X}_{1}=-a X_{1}+a X_{2}+4 X_{4}+X_{5} \\
\dot{X}_{2}=c X_{1}-20 X_{1} X_{3}-X_{2} \\
\dot{X}_{3}=20 X_{1} X_{2}-b X_{3} \\
\dot{X}_{4}=-5 X_{1} X_{3}+p X_{4} \\
\dot{X}_{5}=q X_{1}
\end{array}\right.
$$

in which $X_{1}=\frac{x_{1}}{20}, X_{2}=\frac{x_{2}}{20}, X_{3}=\frac{x_{3}}{20}, X_{4}=\frac{x_{4}}{80}$, and $X_{5}=\frac{x_{5}}{20}$. The schematic of the designed circuit is presented in Fig. 14.

By applying Kirchhoff's laws to the electronic circuit in Fig. 14, its circuital equations are derived in the following form

$$
\left\{\begin{aligned}
\frac{d v_{C_{1}}}{d t} & =-\frac{1}{R_{1} C_{1}} v_{C_{1}}+\frac{1}{R_{2} C_{1}} v_{C_{2}}+\frac{1}{R_{3} C_{1}} v_{C_{4}}+\frac{1}{R_{4} C_{1}} v_{C_{5}} \\
\frac{d v_{C_{2}}}{d t} & =\frac{1}{R_{5} C_{2}} v_{C_{1}}-\frac{1}{10 R_{6} C_{2}} v_{C_{1}} v_{C_{3}}-\frac{1}{R_{7} C_{2}} v_{C_{2}} \\
\frac{d v_{C_{3}}}{d t} & =\frac{1}{10 R_{8} C_{3}} v_{C_{1}} v_{C_{2}}-\frac{1}{R_{9} C_{3}} v_{C_{3}} \\
\frac{d v_{C_{4}}}{d t} & =-\frac{1}{10 R_{10} C_{4}} v_{C_{1}} v_{C_{3}}+\frac{1}{R_{11} C_{4}} v_{C_{4}} \\
\frac{d v_{C_{5}}}{d t} & =\frac{1}{R_{12} C_{5}} v_{C_{1}}
\end{aligned}\right.
$$

where $v_{C_{1}}, v_{C_{2}}, v_{C_{3}}, v_{C_{4}}, v_{C_{5}}$ are the voltages across the capacitors $C_{1}, C_{2}, C_{3}, C_{4}$ and $C_{5}$, respectively. It is noting that there are five operational amplifiers $\left(U_{1}, U_{2}, U_{3}, U_{4}\right.$ and $\left.U_{5}\right)$, which are connected as integrators in Fig. 14. Hence the state variables $X_{1}, X_{2}, X_{3}, X_{4}$, $X_{5}$ of system (55) are the voltages $v_{C_{1}}, v_{C_{2}}, v_{C_{3}}, v_{C_{4}}, v_{C_{5}}$, respectively.

The values of the electronic components in Fig. 14 are chosen to match known parameters of system (5):

$$
\begin{aligned}
& R_{1}=R_{2}=20 \mathrm{k} \Omega, R_{3}=50 \mathrm{k} \Omega, R_{4}=R_{7}=R=200 \mathrm{k} \Omega, R_{5}=7.14 \mathrm{k} \Omega, R_{6}=R_{8}=1 \mathrm{k} \Omega, \\
& R_{9}=75 \mathrm{k} \Omega, R_{10}=4 \mathrm{k} \Omega, R_{11}=153.85 \mathrm{k} \Omega, R_{12}=80 \mathrm{k} \Omega, C_{1}=C_{2}=C_{3}=C_{4}=C_{5}=1 \mathrm{nF}
\end{aligned}
$$



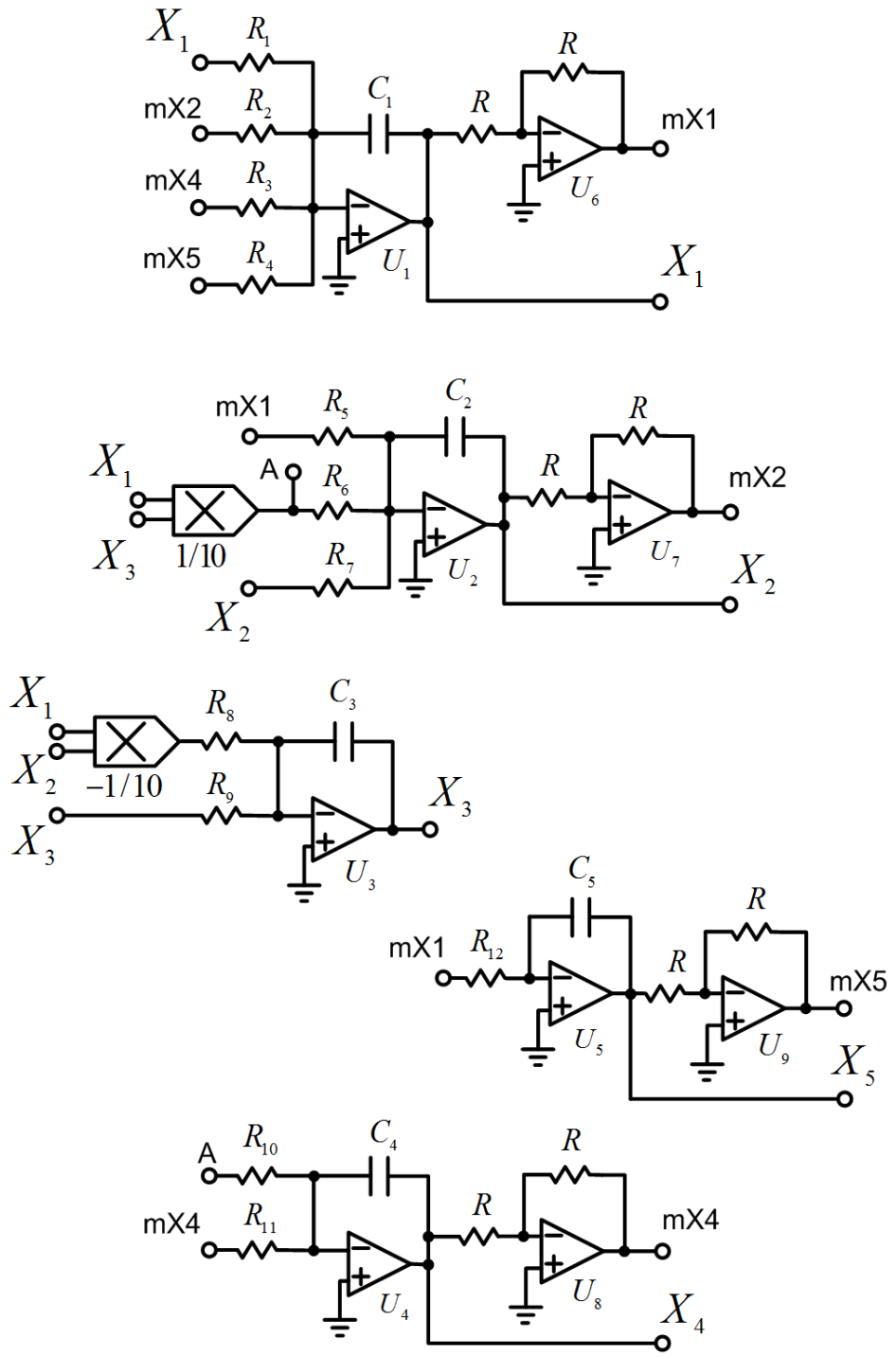

Figure 14: Circuital schematic for realizing novel 5-D hyperchaotic Lorenz system.

The power supplies of all active devices are $\pm 15 V_{D C}$.

The proposed circuit is implemented by using the electronic simulation package Cadence OrCAD. Figs. 15-18 show the obtained phase portraits in $\left(v_{C_{1}}, v_{C_{2}}\right)$-plane, $\left(v_{C_{1}}, v_{C_{3}}\right)$-plane, $\left(v_{C_{1}}, v_{C_{4}}\right)$-plane, and $\left(v_{C_{1}}, v_{C_{5}}\right)$-plane, respectively. 


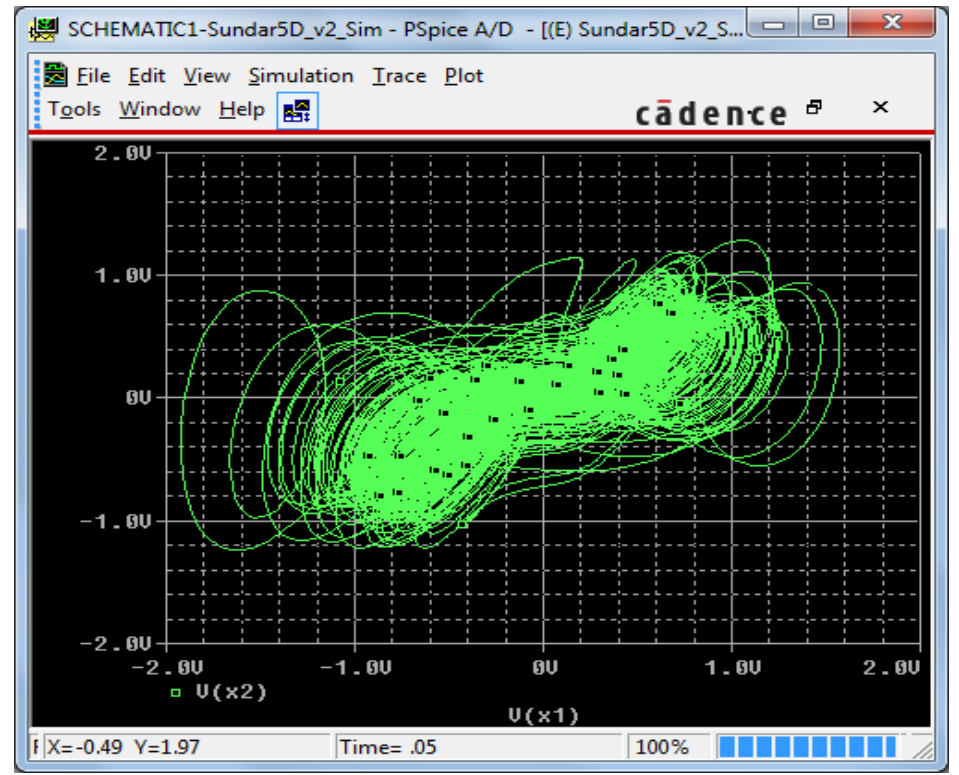

Figure 15: Phase portrait of the designed electronic circuit in $\left(v_{C_{1}}, v_{C_{2}}\right)$-plane obtained from Cadence OrCAD.

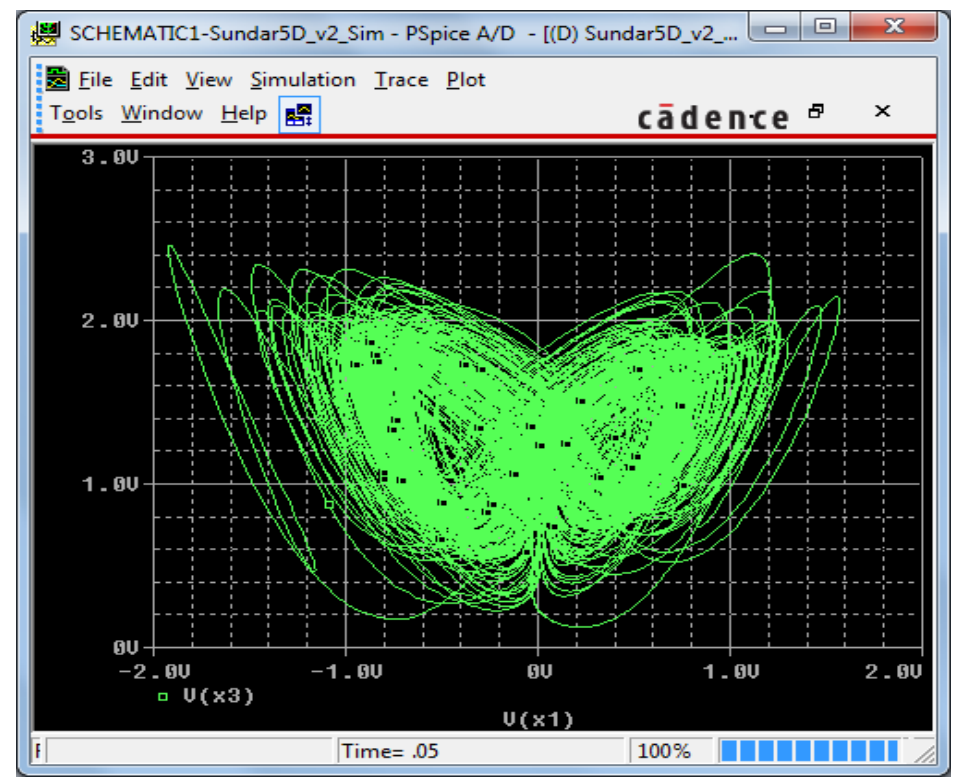

Figure 16: Phase portrait of the designed electronic circuit in $\left(v_{C_{1}}, v_{C_{3}}\right)$-plane obtained from Cadence OrCAD. 
HYPERCHAOS, ADAPTIVE CONTROL AND SYNCHRONIZATION OF A NOVEL 5-D HYPERCHAOTIC SYSTEM WITH THREE POSITIVE LYAPUNOV EXPONENTS AND ITS SPICE IMPLEMENTATION 435

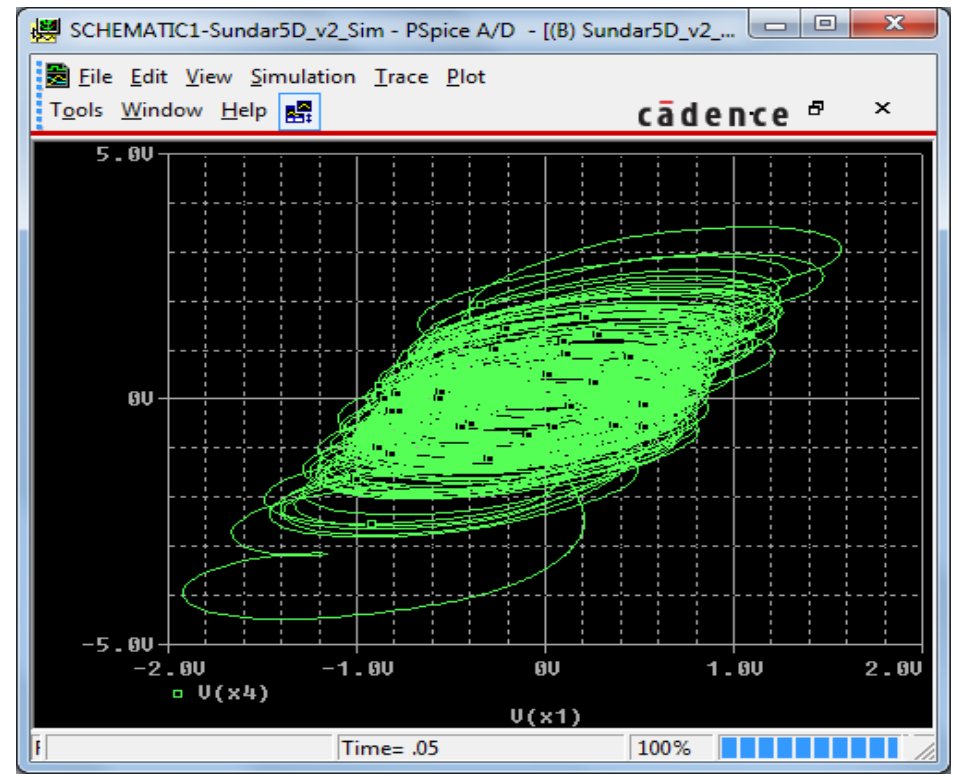

Figure 17: Phase portrait of the designed electronic circuit in $\left(v_{C_{1}}, v_{C_{4}}\right)$-plane obtained from Cadence OrCAD.

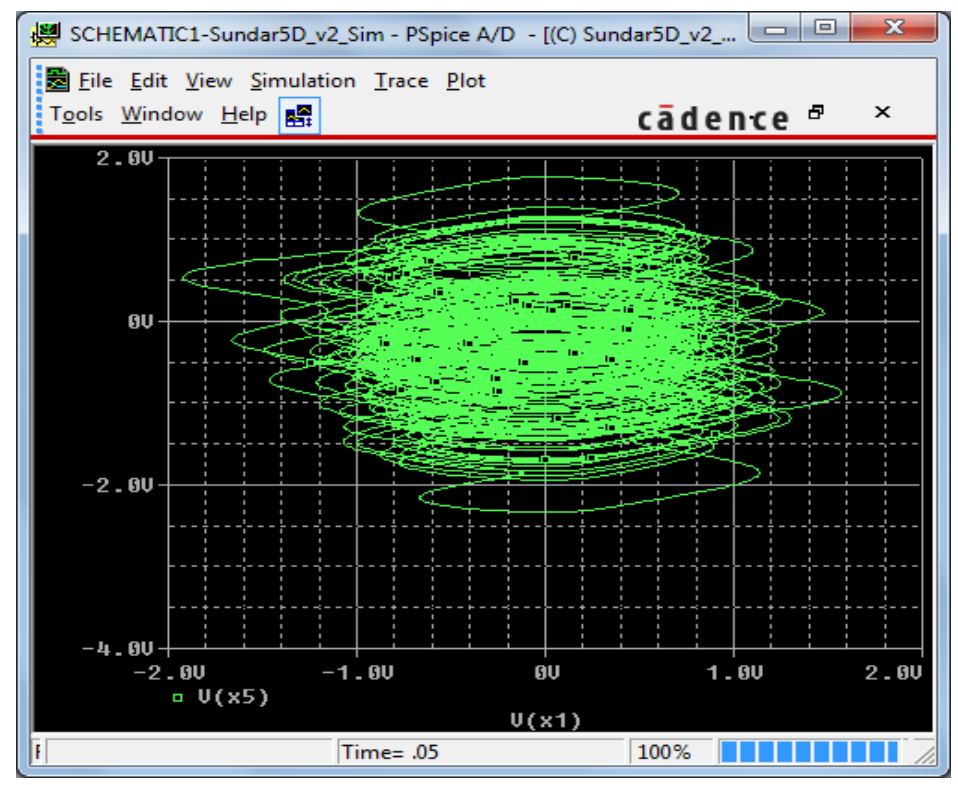

Figure 18: Phase portrait of the designed electronic circuit in $\left(v_{C_{1}}, v_{C_{5}}\right)$-plane obtained from Cadence OrCAD. 


\section{Conclusion}

In the literature, 3-D chaotic systems and 4-D hyperchaotic systems as well as their control and synchronization problems were mainly investigated. However, a 5D system which can generate hyperchaos, especially with three positive Lyapunov exponents, is often rarely reported. In this paper, a twelve-term novel 5-D hyperchaotic Lorenz system with three quadratic nonlinearities has been proposed and its dynamics has been discovered. It is shown that the 5-D hyperchaotic system exhibits three positive Lyapunov exponents and possesses complex dynamical behaviour. In addition, global control and global hyperchaos synchronization of such identical novel 5-D hyperchaotic Lorenz systems with unknown system parameters can be achieved by using an adaptive controller. Moreover, SPICE results obtained from the electronic circuit realization of this novel 5D system show the feasibility of the theoretical introduced model. It is well-known that hyperchaos is better than conventional chaos in a variety of applications. For example, hyperchaos increases the security of chaotic-based communication systems significantly, and in this context, the proposed 5-D hyperchaotic system will be very useful for secure communication systems and other applications as well.

\section{References}

[1] T. KAPITANIAK: Controlling Chaos: Theoretical and Practical Methods in Nonlinear Dynamics. New York, USA, Academic Press, 1996.

[2] A.S. PIKOVSKY, M.G. ROSENBLUM and J. KURTHS: Synchronization: A Unified Concept in Nonlinear Sciences. Cambridge, UK, Cambridge University Press, 2001.

[3] M. LAKSHMANAN and K. MURALI: Chaos in Nonlinear Oscillators: Controlling and Synchronization, Singapore, World Scientific, 1996.

[4] G.L. BAKER and J.P. GOLLUB: Chaotic Dynamics: An Introduction. New York, USA, Cambridge University Press, 1990.

[5] K.T. ALLIGOOD, T.D. SAUER and J.A. YORKE: Chaos: An introduction to Dynamical Systems. New York, Springer-Verlag, 2000.

[6] D. RUELLE and F. TAKENS: On the nature of turbulence. Communications in Mathematical Physics, 20 (1971), 167-192.

[7] R.M. MAY: Limit cycles in predator-prey communities. Science, 177 (1972), 900908.

[8] M.J. FEIGENBAUM: Universal behaviour in nonlinear systems. Physica D: Nonlinear Phenomena, 7 (1983), 16-39. 
HYPERCHAOS, ADAPTIVE CONTROL AND SYNCHRONIZATION OF A NOVEL 5-D HYPERCHAOTIC SYSTEM WITH THREE POSITIVE LYAPUNOV EXPONENTS AND ITS SPICE IMPLEMENTATION 437

[9] E.N. LORENZ: Deterministic nonperiodic flow. Journal of the Atmospheric Sciences, 20 (1963), 130-141.

[10] O.E. RÖSSLER: An equation for continuous chaos. Physics Letters A, 57 (1976), 397-398.

[11] A. ARNEODO, P. COULLET and C. TRESSER: Possible new strange attractors with spiral structure. Communications in Mathematical Physics, 79 (1981), 573579 .

[12] J.C. SPROTT: Some simple chaotic flows. Physical Review E, 50 (1994), 647-650.

[13] G. CHEN and T. UETA: Yet another chaotic attractor. International Journal of Bifurcation and Chaos, 9 (1999), 1465-1466.

[14] J. LÜ and G. CHEN: A new chaotic attractor coined. International Journal of Bifurcation and Chaos, 12 (2002), 659-661.

[15] C.X. LIU, T. LIU, L. LIU and K. LIU: A new chaotic attractor. Chaos, Solitons and Fractals, 22 (2004), 1031-1038.

[16] G. CAI and Z. TAN: Chaos synchronization of a new chaotic system via nonlinear control. Journal of Uncertain Systems, 1 (2007), 235-240.

[17] G. TIGAN and D. OPRIS: Analysis of a 3D chaotic system. Chaos, Solitons and Fractals, 36 (2008), 1315-1319.

[18] D. LI: A three-scroll chaotic attractor. Physics Letters A, 372 (2008), 387-393.

[19] V. SUNDARAPANDIAN and I. PEHLIVAN: Analysis, control, synchronization and circuit design of a novel chaotic system. Mathematical and Computer Modelling, 55 (2012), 1904-1915.

[20] V. SUNDARAPANDIAN: Analysis and anti-synchronization of a novel chaotic system via active and adaptive controllers. Journal of Engineering Science and Technology Review, 6 (2013), 45-52.

[21] S. VAIDYANATHAN: A new six-term 3-D chaotic system with an exponential nonlinearity. Far East Journal of Mathematical Sciences, 79 (2013), 135-143.

[22] S. VAIDYANATHAN: Analysis and adaptive synchronization of two novel chaotic systems with hyperbolic sinusoidal and cosinusoidal nonlinearity and unknown parameters. Journal of Engineering Science and Technology Review, 6 (2013), 53-65.

[23] S. VAIDYANATHAN: A new eight-term 3-D polynomial chaotic system with three quadratic nonlinearities. Far East Journal of Mathematical Sciences, 84 (2014), 219-226. 
[24] S. VAIDYANATHAN: Analysis, control and synchronisation of a six-term novel chaotic system with three quadratic nonlinearities. International Journal of Modelling, Identification and Control, 22 (2014), 41-53.

[25] S. VAIDYANATHAN and K. MADHAVAN: Analysis, adaptive control and synchronization of a seven-term novel 3-D chaotic system. International Journal of Control Theory and Applications, 6 (2013), 121-137.

[26] S. VAIDYANATHAN: Analysis and adaptive synchronization of eight-term 3-D polynomial chaotic systems with three quadratic nonlinearities. European Physical Journal: Special Topics, 223 (2014), 1519-1529.

[27] S. VAIDYANATHAN, C. VOLOS, V.T. PHAM, K. MADHAVAN and B.A. IDOWU: Adaptive backstepping control, synchronization and circuit simulation of a 3-D novel jerk chaotic system with two hyperbolic sinusoidal nonlinearities. Archives of Control Sciences, 24 (2014), 257-285.

[28] S. VAIDYANATHAN: Generalised projective synchronisation of novel 3-D chaotic systems with an exponential non-linearity via active and adaptive control. International Journal of Modelling, Identification and Control, 22 (2014), 207-217.

[29] I. PEHLIVAN, I.M. MOROZ and S. VAIDYANATHAN: Analysis, synchronization and circuit design of a novel butterfly attractor. Journal of Sound and Vibration, 333 (2014), 5077-5096.

[30] S. JAFARI and J.C. SPROTT: Simple chaotic flows with a line equilibrium. Chaos, Solitons and Fractals, 57 (2013), 79-84.

[31] V.T. PHAM, C. VOLOS, S. JAFARI, Z. WEI and X. WANG: Constructing a novel no-equilibrium chaotic system. International Journal of Bifurcation and Chaos, 24 (2014), 1450073.

[32] J.M. TUWANKOTTA: Chaos in a coupled oscillators system with widely spaced frequencies and energy-preserving non-linearity. International Journal of NonLinear Mechanics, 41 (2006), 180-191.

[33] K. SHIMIZU, M. SEKIKAWA and N. INABA: Mixed-mode oscillations and chaos from a simple second-order oscillator under weak periodic perturbation. Physics Letters A, 375 (2011), 1566-1569.

[34] J. KENGNE, J.C. CHEDJOU, G. KENNE and K. KYAMAKYA: Dynamical properties and chaos synchronization of improved Colpitts oscillators. Communications in Nonlinear Science and Numerical Simulation. 17 (2012), 2914-2923.

[35] S. BEHNIA, S. AFRANG, A. AKHSHANI and Kh. MABHOUTI: A novel method for controlling chaos in external cavity semiconductor laser. Optik - International Journal for Light and Electron Optics, 124 (2013), 757-764. 

SYSTEM WITH THREE POSITIVE LYAPUNOV EXPONENTS AND ITS SPICE IMPLEMENTATION 439

[36] E.M. SHAHVERDIEV and K.A. SHORE: Multiplex chaos synchronization in semiconductor lasers with multiple optoelectronic feedbacks. Optik - International Journal for Light and Electron Optics, 124 (2013), 1350-1353.

[37] G. YUAN, X. ZHANG and Z. WANG: Generation and synchronization of feedback-induced chaos in semiconductor ring lasers by injection-locking. Optik - International Journal for Light and Electron Optics, 125 (2014), 1950-1953.

[38] P. GASPARD: Microscopic chaos and chemical reactions. Physica A: Statistical Mechanics and its Applications, 263 (1999), 315-328.

[39] Q.S. LI and R. ZHU: Chaos to periodicity and periodicity to chaos by periodic perturbations in the Belousov-Zhabotinsky reaction. Chaos, Solitons \& Fractals, 19 (2004), 195-201.

[40] M. VILlEGAS, F. AUGUSTIN, A. GILG, A. HMAIDI and U. WEVER: Application of the polynomial chaos expansion to the simulation of chemical reactors with uncertainties. Mathematics and Computers in Simulation, 82 (2012), 805-817.

[41] M. KYRIAZIS: Applications of chaos theory to the molecular biology of aging. Experimental Gerontology, 26 (1991), 569-572.

[42] E. CARLEN, R. CHATELIN, P. DEGOND and B. WENNBERG: Kinetic hierarchy and propagation of chaos in biological swarm models. Physica D: Nonlinear Phenomena, 260 (2013), 90-111.

[43] I. SUÁREZ: Mastering chaos in ecology. Ecological Modelling, 117 (1999), 305314.

[44] J.C. SPROTT, J.A. VANO, J.C. WILDENBERG, M.B. ANDERSON and J.K. NOEL: Coexistence and chaos in complex ecologies. Physics Letters A, 335 (2005), 207-212.

[45] B. SAHOO and S. PORIA: The chaos and control of a food chain model supplying additional food to top-predator. Chaos, Solitons \& Fractals, 58 (2014), 52-64.

[46] K. AIHIRA, T. TAKABE and M. TOYODA: Chaotic neural networks. Physics Letters A, 144 (1990), 333-340.

[47] G. HE, Z. CAO, P. ZHU and H. OGURA: Controlling chaos in a chaotic neural network. Neural Networks, 16 (2003), 1195-1200.

[48] W.Z. HUANG and Y. HUANG: Chaos of a new class of Hopfield neural networks. Applied Mathematics and Computation, 206 (2008), 1-11.

[49] Y. SUN, V. BABOVIC and E.S. CHAN: Multi-step-ahead model error prediction using time-delay neural networks combined with chaos theory. Journal of Hydrology, 395 (2010), 109-116. 
[50] S. LANKALAPALLI and A. GHOSAL: Chaos in robot control equations. International Journal of Bifurcation and Chaos, 7 (1997), 707-720.

[51] Y. NAKAMURA and A. SEKIGUCHI: The chaotic mobile robot. IEEE Transactions on Robotics and Automation, 17 (2001), 898-904.

[52] M. ISLAM and K. MURASE: Chaotic dynamis of a behavior-based miniature mobile robot: effects of environment and control structure. Neural Networks, 18 (2005), 123-144.

[53] H.T. YAU and C.S. SHIEH: Chaos synchronization using fuzzy logic controller. Nonlinear Analysis: Real World Applications, 9 (2008), 1800-1810.

[54] N.S. PAI, H.T. YAU and C.L. KUO: Fuzzy logic combining controller design for chaos control of a rod-type plasma torch system. Expert Systems with Applications, 37 (2010), 8278-8283.

[55] Z. SHI, S. HONG and K. CHEN: Experimental study on tracking the state of analog Chua's circuit with particle filter for chaos synchronization. Physics Letters A, 372 (2008), 5575-5580.

[56] A.E. MATOUK and H.N. AGIZA: Bifurcations, chaos and synchronization in ADVP circuit with parallel resistor. Journal of Mathematical Analysis and Applications, 341 (2008), 259-269.

[57] A.E. MATOUK: Chaos, feedback control and synchronization of a fractional-order modified autonomous Van der Pol-Duffing circuit. Communications in Nonlinear Science and Numerical Simulation, 16 (2011), 975-986.

[58] J.C. SPROTT: Elegant Chaos. Singapore, World Scientific, 2010.

[59] C. LI, X. LIAO and K.W. WONG: Lag synchronization of hyperchaos with application to secure communications. Chaos, Solitons and Fractals, 23 (2005), 183193.

[60] N. SMAOUI, A. KAROUMA and M. ZRIBI: Secure communications based on the synchronization of the hyperchaotic Chen and the unified chaotic systems. Communications in Nonlinear Science and Numerical Simulation, 16 (2011), 3279-3293.

[61] X.J. WU, H. WANG and H.T. LU: Hyperchaotic secure communication via generalized function projective synchronization. Nonlinear Analysis: Real World Applications, 12 (2011), 1288-1299.

[62] T.I. CHIEN and T.L. LIAO: Design of secure digital communication systems using chaotic modulation, cryptography and chaotic synchronization. Chaos, Solitons \& Fractals, 24 (2005), 241-245. 
HYPERCHAOS, ADAPTIVE CONTROL AND SYNCHRONIZATION OF A NOVEL 5-D HYPERCHAOTIC SYSTEM WITH THREE POSITIVE LYAPUNOV EXPONENTS AND ITS SPICE IMPLEMENTATION 441

[63] B. ZHANG, M. CHEN and D. ZHOU: Chaotic secure communication based on particle filtering. Chaos, Solitons \& Fractals, 30 (2006), 1273-1280.

[64] X. WU, C. BAI and H. KAN: A new color image cryptosystem via hyperchaos synchronization. Communications in Nonlinear Science and Numerical Simulation, 19 (2014), 1884-1897.

[65] Q. ZHANG, L. GUO and X. WEI: A novel image fusion encryption algorithm based on DNA sequence operation and hyper-chaotic system. Optik - International Journal for Light and Electron Optics, 124 (2013), 3596-3600.

[66] G. YE and J. ZHOU: A block chaotic image encryption scheme based on selfadaptive modelling. Applied Soft Computing, 22 (2014), 351-357.

[67] H. LIU, X. WANG and A. KADIR: Color image encryption using Choquet fuzzy integral and hyper chaotic system. Optik - International Journal for Light and Electron Optics, 124 (2013), 3257-3533.

[68] A. BUSCARINO, L. FORTUNA and M. FRASCA: Experimental robust synchronization of hyperchaotic circuits. Physica D: Nonlinear Phenomena, 238 (2009), 1917-1922.

[69] N. YUJUN, W. XINGYUAN, W. MINGJUN and Z. HUAGUANG: A new hyperchaotic system and its circuit implementation. Communications in Nonlinear Science and Numerical Simulation, 15 (2010), 3518-3524.

[70] X. WEI, F. YUNFEI and L. QIANG: A novel four-wing hyper-chaotic system and its circuit implementation. Procedia Engineering, 29 (2012), 1264-1269.

[71] P. ZHOU and K. HUANG: A new 4-D non-equilibrium fractional-order chaotic system and its circuit implementation. Communications in Nonlinear Science and Numerical Simulation, 19 (2014), 2005-2011.

[72] O.E. RÖSSLER: An equation for hyperchaos. Physics Letters A, 71 (1979), 155157.

[73] Q. JIA: Hyperchaos generated from the Lorenz chaotic system and its control. Physics Letters A, 366 (2007), 217-222.

[74] A. CHEN, J. LU, J.LÜ and S. YU: Generating hyperchaotic Lü attractor via state feedback control. Physica A, 364 (2006), 103-110.

[75] X. LI: Modified projective synchronization of a new hyperchaotic system via nonlinear control. Communications in Theoretical Physics, 52 (2009), 274-278.

[76] J. WANG and Z. CHEN: A novel hyperchaotic system and its complex dynamics. International Journal of Bifurcation and Chaos, 18 (2008), 3309-3324. 
[77] D. GHOSH and S. BHATTACHARYA: Projective synchronization of new hyperchaotic system with fully unknown parameters. Nonlinear Dynamics, 61 (2010), 11-21.

[78] S. VAIDYANATHAN: A ten-term novel 4-D hyperchaotic system with three quadratic nonlinearities and its control. International Journal of Control Theory and Applications, 6 (2013), 97-109.

[79] G. HU: Generating hyperchaotic attractors with three positive Lyapunov exponents via state feedback control. International Journal of Bifurcation and Chaos, 19 (2009), 651.

[80] Q. YANG and C. CHEN: A 5D hyperchaotic system with three positive Lyapunov exponents coined. International Journal of Bifurcation and Chaos, 23 (2013), 1350109.

[81] Q. JIA: Hyperchaos generated from the Lorenz chaotic system and its control. Physics Letters A, 366 (2007), 217-222.

[82] V. SUNDARAPANDIAN: Output regulation of the Lorenz attractor. Far East Journal of Mathematical Sciences, 42 (2010), 289-299.

[83] S. VAIDYANATHAN: Output regulation of Arneodo-Coullet chaotic system. Communications in Computer and Information Science, 131 (2011), 585-593.

[84] S. VAIDYANATHAN: Output regulation of the unified chaotic system. Communications in Computer and Information Science, 198 (2011), 1-9.

[85] S. VAIDYANATHAN: Output regulation of the Liu chaotic system. Applied Mechanics and Materials, 110 (2012), 3982-3989.

[86] G. CHEN: A simple adaptive feedback control method for chaos and hyper-chaos control. Applied Mathematics and Computation, 217 (2011), 7258-7264.

[87] S. VAIDYANATHAN: Adaptive controller and synchronizer design for the QiChen chaotic system. Lecture Notes of the Institute for Computer Sciences, SocialInformatics and Telecommunication Engineering, 85 (2012), 124-133.

[88] V. SUNDARAPANDIAN: Adaptive control and synchronization design for the LuXiao chaotic system. Lecture Notes on Electrical Engineering, 131 (2013), 319327.

[89] S. VAIDYANATHAN: Analysis, control and synchronization of hyperchaotic Zhou system via adaptive control. Advances in Intelligent Systems and Computing, 177 (2013), 1-10. 
HYPERCHAOS, ADAPTIVE CONTROL AND SYNCHRONIZATION OF A NOVEL 5-D HYPERCHAOTIC SYSTEM WITH THREE POSITIVE LYAPUNOV EXPONENTS AND ITS SPICE IMPLEMENTATION 443

[90] J. ZHENG: A simple universal adaptive feedback controller for chaos and hyperchaos control. Computers \& Mathematics with Applications, 61 (2011), 20002004.

[91] W. LIN: Adaptive chaos control and synchronization in only locally Lipschitz systems. Physics Letters A, 372 (2008), 3195-3200.

[92] D.Q. WEI, X.S. LUO, B. ZHANG and Y.H. QIN: Controlling chaos in spaceclamped FitzHugh-Nagumo neuron by adaptive passive method. Nonlinear Analysis: Real World Applications, 11 (2010), 1752-1759.

[93] M.T. YASSEN: Chaos control of chaotic dynamical systems using backstepping design. Chaos, Solitons \& Fractals, 27 (2006), 537-548.

[94] J.A. LAOYE, U.E. VINCENT and S.O. KAREEM: Chaos control of 4D chaotic systems using recursive backstepping nonlinear controller. Chaos, Solitons \& Fractals, 39 (2009), 356-362.

[95] D. LIN, X. WANG, F. NIAN and Y. ZHANG: Dynamic fuzzy neural networks modeling and adaptive backstepping tracking control of uncertain chaotic systems. Neurocomputing, 73 (2010), 2873-3881.

[96] S. VAIDYANATHAN: Sliding mode control based global chaos control of Liu-LiuLiu-Su chaotic system. International Journal of Control Theory and Applications, 5 (2012), 15-20.

[97] S. VAIDYANATHAN: Global chaos control of hyperchaotic Liu system via sliding mode control. International Journal of Control Theory and Applications, 5 (2012), $117-123$.

[98] H. FUJISAKA and T. YAMADA: Stability theory of synchronized motion in coupled-oscillator systems. Progress of Theoretical Physics, 69, 32-47, (1983).

[99] L.M. PECORA and T.L. CAROLL: Synchronization in chaotic systems. Physical Review Letters, 64, 821-825, (1990).

[100] V. SUNDARAPANDIAN and R. KARTHIKEYAN: Anti-synchronization of hyperchaotic Lorenz and hyperchaotic Chen systems by adaptive control. International Journal of Signal System Control and Engineering Application, 4, 18-25, (2011).

[101] S. VAIDYANATHAN: Anti-synchronization of Sprott-I and Sprott-M chaotic systems via adaptive control. International Journal of Control Theory and Applications, 5, 41-59, (2012).

[102] V. SUNDARAPANDIAN and R. KARTHIKEYAN: Anti-synchronization of Lu and Pan chaotic systems by adaptive nonlinear control. International Journal of Soft Computing, 6, 111-118, (2011). 
[103] V. SUNDARAPANDIAN and R. KARTHIKEYAN: Hybrid synchronization of hyperchaotic Lorenz and hyperchaotic Chen systems via active control. Journal of Engineering and Applied Sciences, 7, 254-264, (2012).

[104] R. KARTHIKEYAN and V. SUNDARAPANDIAN: Hybrid chaos synchronization of four-scroll systems via active control. Journal of Electrical Engineering, 65, 97-103, (2014).

[105] M.G. ROSENBLUM, A.S. PIKOVSKY and J. KURTHS: From phase to lag synchronization in coupled chaotic oscillators. Physical Review Letters, 78, 41934196, (1997).

[106] M.G. ROSENBLUM, A.S. PIKOVSKY and J. KURTHS: Phase synchronization of chaotic oscillators. Physical Review Letters, 76, 1804-1807, (1996).

[107] V. ASTAKHOV, A. SHABUNIN and V. ANISHCHENKO: Antiphase synchronization in symmetrically coupled self-oscillators. International Journal of Bifurcation and Chaos, 10, 849-857, (2000).

[108] N.F. RULKOV, M.M. SUSHCHIK, L.S. TSIMRING and H.D.I. ABABARNEL: Generalized synchronization of chaos in directionally coupled chaotic systems. Physical Review E, 51, 980-994, (1995).

[109] R. MAINIERI and J. REHACEK: Projective synchronization in threedimensional chaotic system. Physical Review Letters, 82, 3042-3045, (1999).

[110] P. SARASU and V. SUNDARAPANDIAN: The generalized projective synchronization of hyperchaotic Lorenz and hyperchaotic Qi systems via active control. International Journal of Soft Computing, 6, 216-223, (2011).

[111] P. SARASU and V. SUNDARAPANDIAN: Adaptive controller design for the generalized projective synchronization of 4-scroll systems. International Journal of Signal System Control and Engineering Application, 5, 21-30, (2012).

[112] S. VAIDYANATHAN and S. PAKIRISWAMY: Generalized projective synchronization of six-term Sundarapandian chaotic systems by adaptive control. International Journal of Control Theory and Applications, 6, 153-163, (2013).

[113] H.N. AGIZA and M.T. YASSEN: Synchronization of Rossler and Chen chaotic dynamical systems using active control. Physics Letters A, 278, 191-197, (2001).

[114] U.E. VINCENT: Synchronization of identical and non-identical 4-D chaotic systems using active control. Chaos, Solitons and Fractals, 37, 1065-1075, (2008).

[115] B.A. IDOWU, U.E. VINCENT and A.N. NJAH: Synchronization of chaos in nonidentical parametrically excited systems. Chaos, Solitons and Fractals, 39, 23222331, (2009). 
HYPERCHAOS, ADAPTIVE CONTROL AND SYNCHRONIZATION OF A NOVEL 5-D HYPERCHAOTIC SYSTEM WITH THREE POSITIVE LYAPUNOV EXPONENTS AND ITS SPICE IMPLEMENTATION 445

[116] S. VAIDYANATHAN and K. RAJAGOPAL: Hybrid synchronization of hyperchaotic Wang-Chen and hyperchaotic lorenz systems by active non-linear control. International Journal of Signal System Control and Engineering Application, 4, 55-61, (2011).

[117] V. SUNDARAPANDIAN and R. KARTHIKEYAN: Anti-synchronization of Lü and Pan chaotic systems by adaptive nonlinear control. European Journal of Scientific Research, 64, 94-106, (2011).

[118] V. SUNDARAPANDIAN and R. KARTHIKEYAN: Adaptive antisynchronization of Uncertain Tigan and Li Systems. Journal of Engineering and Applied Sciences, 7, 45-52, (2012).

[119] P. SARASU and V. SUNDARAPANDIAN: Generalized projective synchronization of three-scroll chaotic systems via adaptive control. European Journal of Scientific Research, 72, 504-522, (2012).

[120] S. VAIDYANATHAN and K. RAJAGOPAL: Global chaos synchronization of hyperchaotic Pang and hyperchaotic Wang systems via adaptive control. International Journal of Soft Computing, 7, 28-37, (2012).

[121] X. TAN, J. ZHANG and Y. YANG: Synchronizing chaotic systems using backstepping design. Chaos, Solitons and Fractals, 16, 37-45, (2003).

[122] S. RASAPPAN and S. VAIDYANATHAN: Global chaos synchronization of WINDMI and Coullet chaotic systems by backstepping control. Far East Journal of Mathematical Sciences, 67, 265-287, (2012).

[123] S. RASAPPAN and S. VAIDYANATHAN: Hybrid synchronization of $n$-scroll Chua circuits using adaptive backstepping control design with recursive feedback. Malaysian Journal of Mathematical Sciences, 7, 219-226, (2013).

[124] R. SURESH and V. SUNDARAPANDIAN: Global chaos synchronization of a family of $n$-scroll hyperchaotic Chua circuits using backstepping control with recursive feedback. Far East Journal of Mathematical Sciences, 73, 73-95, (2013).

[125] S. RASAPPAN and S. VAIDYANATHAN: Global chaos synchronization of WINDMI and Coullet chaotic systems using adaptive backstepping control design. Kyungpook Mathematical Journal, 54, 293-320, (2014).

[126] S. VAIDYANATHAN and S. RASAPPAN: Global chaos synchronization of $n$ scroll Chua circuit and Lur'e system using backstepping control design with recursive feedback. Arabian Journal for Science and Engineering, 39, 3351-3364, (2014). 
[127] D. ZHANG and J. XU: Projective synchronization of different chaotic timedelayed neural networks based on integral sliding mode controller. Applied Mathematics and Computation, 217, 164-174, (2010).

[128] V. SUNDARAPANDIAN and S. SIVAPERUMAL: Sliding controller design of hybrid synchronization of four-wing chaotic systems. International Journal of Soft Computing, 6, 224-231, (2011).

[129] S. VAIDYANATHAN: Global chaos control of hyperchaotic Liu system via sliding control method. International Journal of Control Theory and Applications, 5, 117-123, (2012).

[130] S. VAIDYANATHAN and S. SAMPATH: Anti-synchronization of four-wing chaotic systems via sliding mode control. International Journal of Automatic Computing, 9, 274-279, (2012).

[131] S. VAIDYANATHAN: Global chaos synchronisation of identical Li-Wu chaotic systems via sliding mode control. International Journal of Modelling, Identification and Control, 22 (2014), 170-177. 